\title{
Nonlocal-to-Local Convergence of Cahn-Hilliard Equations: Neumann Boundary Conditions and Viscosity Terms
}

\author{
Elisa Davoli@, Luca Scarpa@ \& Lara Trussardi
}

Communicated by F. LIN

\begin{abstract}
We consider a class of nonlocal viscous Cahn-Hilliard equations with Neumann boundary conditions for the chemical potential. The double-well potential is allowed to be singular (e.g. of logarithmic type), while the singularity of the convolution kernel does not fall in any available existence theory under Neumann boundary conditions. We prove well-posedness for the nonlocal equation in a suitable variational sense. Secondly, we show that the solutions to the nonlocal equation converge to the corresponding solutions to the local equation, as the convolution kernels approximate a Dirac delta. The asymptotic behaviour is analyzed by means of monotone analysis and Gamma convergence results, both when the limiting local Cahn-Hilliard equation is of viscous type and of pure type.
\end{abstract}

\section{Contents}

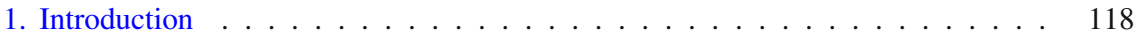

2. Mathematical Setting . . . . . . . . . . . . . . . . . . 123

2.1. Assumptions . . . . . . . . . . . . . . . . . . . . . . . . 123

2.2. Variational Setting and Preliminaries . . . . . . . . . . . . . . . . 124

3. Main Results . . . . . . . . . . . . . . . . . . . . . . . . . . . . . . . . . . . 129

4. Proof of Theorem $3.2 \ldots \ldots \ldots \ldots$

4.1. Approximation . . . . . . . . . . . . . . . . . . . 132

4.2. Uniform Estimates ． . . . . . . . . . . . . . . . . . . . . . . . 133

4.3. Passage to the Limit as $\lambda \searrow 0 \ldots \ldots$. . . . . . . . . . . . . . . 135

4.4. Continuous Dependence . . . . . . . . . . . . . . . . . . . . 139

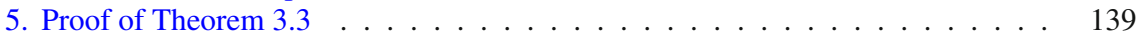

5.1. The Case $\tau>0 \ldots \ldots \ldots$. . . . . . . . . . . . . . . . . . . . . . 139

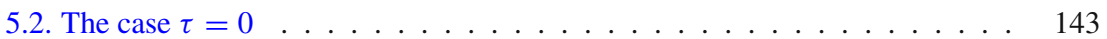

References . . . . . . . . . . . . . . . . . . . 146 


\section{Introduction}

The aim of the present paper is to study the well-posedness and the asymptotic behaviour as $\varepsilon \searrow 0$ of a family of nonlocal viscous Cahn-Hilliard equations with Neumann boundary conditions in the following form:

$$
\begin{aligned}
\partial_{t} u_{\varepsilon}-\Delta \mu_{\varepsilon}=0 & \text { in }(0, T) \times \Omega, \\
\mu_{\varepsilon}=\tau_{\varepsilon} \partial_{t} u_{\varepsilon}+\left(K_{\varepsilon} * 1\right) u_{\varepsilon}-K_{\varepsilon} * u_{\varepsilon}+\Psi^{\prime}\left(u_{\varepsilon}\right)-g_{\varepsilon} & \text { in }(0, T) \times \Omega, \\
\partial_{\mathbf{n}} \mu_{\varepsilon}=0 & \text { on }(0, T) \times \partial \Omega, \\
u_{\varepsilon}(0)=u_{0, \varepsilon} & \text { in } \Omega,
\end{aligned}
$$

where $\Omega$ is a smooth bounded domain in $\mathbb{R}^{d}(d=2,3), T>0$ is a fixed final time, and $\Psi^{\prime}$ represents the derivative a double-well potential. Moreover, $\varepsilon>0$ is a fixed parameter, $\tau_{\varepsilon}>0$ is a positive viscosity coefficient, $K_{\varepsilon}: \Omega \times \Omega \rightarrow \mathbb{R}$ is a suitable symmetric convolution kernel, and $g_{\varepsilon}$ represents a distributed forcing term. The variables $u_{\varepsilon}$ and $\mu_{\varepsilon}$ are referred to as "order parameter" and "chemical potential", respectively.

The evolution problem (1.1)-(1.4) is related to the gradient flow (in the $H^{-1}$ metric) associated to a nonlocal free energy functional of the form

$$
\mathcal{E}_{\varepsilon}(\varphi)=\frac{1}{4} \int_{\Omega} \int_{\Omega} K_{\varepsilon}(x, y)|\varphi(x)-\varphi(y)|^{2} \mathrm{~d} x \mathrm{~d} y+\int_{\Omega} \Psi(\varphi(x)) \mathrm{d} x .
$$

Indeed, the contributions $\left(K_{\varepsilon} * 1\right) u_{\varepsilon}-K_{\varepsilon} * u_{\varepsilon}+\Psi^{\prime}\left(u_{\varepsilon}\right)$ in the definition of the chemical potential are obtained exactly from the (sub)differentiation of the functional (1.5). The extra term $\tau_{\varepsilon} \partial_{t} u_{\varepsilon}$ represents on the other side a viscosity regularization, acting on the dissipation of the system.

The analysis of nonlocal models dates back to the early 90's, when Giacomin and Lebowitz investigated, in their seminal paper [36], a hydrodynamic limit of a microscopic model for a $d$-dimensional lattice gas evolving via a Poisson nearestneighbor process. In that work, the authors derived a free energy functional in nonlocal form (1.5), and proposed the corresponding gradient flow to model phasechange in binary alloys. The viscous regularization in the definition of the chemical potential was originally introduced in the context of the local Cahn-Hilliard equation by Novick-Cohen in [50]. The mathematical literature on the nonlocal Cahn-Hilliard equation is widely developed: we can mention, among many others, the contributions $[2,5,27,28,39]$ and the references therein.

The rapidly growing attention to the nonlocal Cahn-Hilliard equation is due on the one hand to its microscopic justification, and on the other hand to its connection with the corresponding local model. Indeed, at least in a formal way, the nonlocal dynamics approach the local ones when the family of interaction kernels $\left(K_{\varepsilon}\right)_{\varepsilon}$ concentrates around the origin. The main issue we assess in this paper is the asymptotic convergence of solutions to the nonlocal system (1.1)-(1.4) to the corresponding local one, as the data $\left(g_{\varepsilon}\right)_{\varepsilon}$ approximate a new source $g$ and the coefficients $\tau_{\varepsilon}$ converge to a certain new viscosity parameter $\tau$. The local form of 
the limiting Cahn-Hilliard equation reads is

$$
\begin{aligned}
\partial_{t} u-\Delta \mu=0 & \text { in }(0, T) \times \Omega, \\
\mu=\tau \partial_{t} u-\Delta u+\Psi^{\prime}(u)-g & \text { in }(0, T) \times \Omega, \\
\partial_{\mathbf{n}} u=0 \text { and } \partial_{\mathbf{n}} \mu=0 & \text { on }(0, T) \times \partial \Omega, \\
u(0)=u_{0} & \text { in } \Omega,
\end{aligned}
$$

where $\tau \geqq 0$ is the limiting viscosity parameter, which is allowed to vanish. The choices $\tau>0$ and $\tau=0$ correspond to the viscous case and pure case, respectively.

As its nonlocal counterpart, the local Cahn-Hilliard equation is related to the gradient flow in the $H^{-1}$ metric of the Ginzburg-Landau free energy functional

$$
\mathcal{E}(\varphi)=\frac{1}{2} \int_{\Omega}|\nabla \varphi(x)|^{2} \mathrm{~d} x+\int_{\Omega} \Psi(\varphi(x)) \mathrm{d} x,
$$

in the sense that the contribution $-\Delta u+\Psi^{\prime}(u)$ results from the subdifferentiation of $\mathcal{E}$. Again, the viscosity term $\tau \partial_{t} u$ acts on the dissipation of the system: if $\tau=0$, one recovers the so-called pure Cahn-Hilliard equation, while if $\tau>0$ one obtains the viscous Cahn-Hilliard equation. In our analysis, the nonlocal viscosity coefficients $\left(\tau_{\varepsilon}\right)_{\varepsilon}$ are assumed to be strictly positive, while the local coefficient $\tau$ is allowed to vanish.

The local Cahn-Hilliard equation was first proposed in [9] in relation to phasechange in metallic alloys and to spinodal decomposition (see [44]). Nowadays, the model is a widely used in various contexts such as diffuse interface modelling in physics and biology, with several applications to tumor growth dynamics, image processing, and population dynamics. From the mathematical point of view, the local Cahn-Hilliard equation has been studied thoroughly in the last decades, also in much more complex settings. We mention, among many others, the works [11$13,15,16,18,37,43]$ on well-posedness also under more general dynamic boundary conditions. Some studies on nonlinear viscosity contributions have been proposed in $[6,49,56]$. We also recall the contributions $[14,19,20,23,40]$ dealing with optimal control problems, as well as $[17,22,38]$ on asymptotics. The local Cahn-Hilliard equation has also been widely studied recently in connection to diffuse-interface models for fluid-dynamics: we refer to $[1,3,10,29,30]$ and the references therein.

As has already been mentioned, the behaviour of the nonlocal Cahn-Hilliard equation "approaches" the one of the local equation when the family of convolution kernels is sufficiently peaked around 0 . The study of nonlocal-to-local convergence of energy functionals in relation to Sobolev spaces theory had been carried out originally by by Bourgain et al. [7,8], and by Mazy'a and Shaposhnikova $[45,46]$. This asymptotic analysis was also extended by Ponce [51,52], with studies on Gamma convergence and nonlocal Poincaré-type inequalities. A first criterion for the convergence of gradient flows from the Gamma-convergence of the respective energies was given by Sandier and Serfaty [55] in a abstract setting and for smooth energies, with applications to Ginzburg-Landau functionals (see also [42,53,57] for further details in this direction). 
In particular, the above-mentioned results $[51,52]$ provide the pointwise convergence

$$
\lim _{\varepsilon \searrow 0} \mathcal{E}_{\varepsilon}(\varphi)=\mathcal{E}(\varphi) \quad \forall \varphi \in H^{1}(\Omega)
$$

as soon as the convolution kernels $\left(K_{\varepsilon}\right)_{\varepsilon}$ are chosen as

$$
K_{\varepsilon}: \Omega \times \Omega \rightarrow[0,+\infty), \quad K_{\varepsilon}(x, y):=\frac{\rho_{\varepsilon}(|x-y|)}{|x-y|^{2}}, \quad x, y \in \Omega,
$$

where $\left(\rho_{\varepsilon}\right)_{\varepsilon}$ is a suitable family of mollifiers converging to a Dirac delta.

Building upon these variational convergences, in a previous contribution of ours [24] we rigorously derived some nonlocal-to-local asymptotics of solutions to Cahn-Hilliard equations in the setting of periodic boundary conditions and with no viscosity effects. The periodic setting adopted in [24] was fundamental to overcome the singular behaviour of the convolution kernel (1.11). Indeed, kernels in the form (1.11) do not possess any $W^{1,1}$ regularity (see for example [21, Remark 1]), which is the usual minimum requirement in the whole literature on nonlocal Cahn-Hilliard systems. This resulted in the impossibility of framing the nonlocal problem in any available existence theory, and required an ad-hoc analysis. In this direction, the arguments strongly relied on the assumption of periodic boundary conditions.

The results in [24] (see also [47] for a simpler case) are very satisfactory since they provide a novel contribution in the direction of local asymptotics of CahnHilliard equations. Nevertheless, the most natural choice of boundary conditions in phase-field modelling if of no-flux type. Consequently, it is crucial in this direction to generalize the periodic framework to other settings more suited for applications. The nonlocal-to-local convergence of pure Cahn-Hilliard equations with Neumann boundary conditions was, to the authors' knowledge, still an open problem. The main novelty of the present paper is to finally extend some rigorous nonlocal-tolocal convergence results for Cahn-Hilliard equations to the case of homogeneous Neumann boundary conditions.

Let us briefly describe now the main difficulties arising in the case of Neumann boundary conditions. The first hurdle has been already anticipated and concerns the regularity of the convolution kernel. Indeed, in the form (1.11) the kernel $K_{\varepsilon}$ is not $W^{1,1}$, and not even $L^{1}$ in dimension $d=2$. This results in the necessity of rigorously formulate the nonlocal problem without relying on any available existence theory. The main idea here is that even if the convolution operator $\varphi \mapsto K_{\varepsilon} * \varphi$ may be ill-defined under (1.11), the nonlocal operator $B_{\varepsilon}: \varphi \mapsto\left(K_{\varepsilon} * 1\right) \varphi-\left(K_{\varepsilon} * \varphi\right)$ appearing in the equation (1.2) can be rigorously defined instead.

The second main problem consists in the (im)possibility of proving space regularity for the solutions to the nonlocal equation (i.e. when $\varepsilon>0$ is fixed). If the convolution kernel is $W^{1,1}$ this follows directly from the properties of the convolution, i.e. formally shifting the gradient operator on the kernel as $\nabla\left(K_{\varepsilon} * u_{\varepsilon}\right)=\left(\nabla K_{\varepsilon}\right) * u_{\varepsilon}$. However, for singular kernels as in (1.11) this procedure fails. Under periodic boundary conditions (i.e. working on the $d$-dimensional flat torus) the main idea to overcome this problem was to use a certain integration-by-parts formula, which hinges in turn on some compatibility conditions between the convolution operator 
and the Laplace operator. More specifically, in [24] the periodic setting allowed to prove a (formal) relation in the form $\nabla\left(K_{\varepsilon} * u_{\varepsilon}\right)=K_{\varepsilon} * \nabla u_{\varepsilon}$, from which one could deduce $H^{1}$-regularity of the nonlocal solutions. Nevertheless, under Neumann boundary conditions (i.e. working on a bounded domain $\Omega \subset \mathbb{R}^{d}$ ), in order to prove an analogous compatibility relation one is forced to extend the nonlocal solution $u_{\varepsilon}$ to 0 outside $\Omega$. Clearly, $H^{1}$-regularity in $\Omega$ does not imply $H^{1}$-regularity on the whole $\mathbb{R}^{d}$ for such extension. This gives rise to several extra boundary contribution terms which blow up as the approximating parameter vanishes.

The main consequence is that in the case of Neumann boundary conditions one loses any $H^{1}$-estimate on the nonlocal solutions. It follows that the natural variational setting to frame the nonlocal problem (1.1)-(1.4) is not the usual one given by the triple $\left(H^{1}(\Omega), L^{2}(\Omega), H^{1}(\Omega)^{*}\right)$, but instead an abstract one $\left(V_{\varepsilon}, L^{2}(\Omega), V_{\varepsilon}^{*}\right)$, depending on $\varepsilon$, where $V_{\varepsilon}$ represents, roughly speaking, the domain of the nonlocal energy contribution in (1.5). As the inclusion $V_{\varepsilon} \hookrightarrow L^{2}(\Omega)$ is not compact, one loses any reasonable compactness property on the approximated solutions in order to pass to the limit in the nonlinearity. This issue is overcome by the introduction of the viscosity term $\tau_{\varepsilon} \partial_{t} u_{\varepsilon}$. Indeed, if $\tau_{\varepsilon}$ is strictly positive one can show "by hand" a strong convergence in $L^{2}(\Omega)$ for some regularized solutions, even without relying on any $H^{1}$ estimates.

The third main problem concerns the boundary conditions of Neumann type for $u$ in the limiting local problem. Indeed, while the nonlocal system is of order 2 in space, hence it only needs one boundary condition (for the chemical potential), the limiting local equation is of order 4 in space and requires two boundary conditions instead: one for $\mu$ and one for $u$. One of the major point is to understand which is the natural extra boundary condition for $u$, and how this one emerges when $\varepsilon \searrow 0$. It is clear that the Neumann boundary condition for the chemical potential is preserved by the local asymptotics. On the other hand, the scenario for $u$ is more subtle: the answer is implicitly given by studying the Gamma convergence of the nonlocal energies. Indeed, in [52] Ponce proved a Gamma convergence result in the form

$$
\begin{gathered}
\lim _{\varepsilon \searrow 0} \frac{1}{4} \int_{\Omega} \int_{\Omega} K_{\varepsilon}(x, y)\left|\varphi_{\varepsilon}(x)-\varphi_{\varepsilon}(y)\right|^{2} \mathrm{~d} x \mathrm{~d} y \\
\quad= \begin{cases}\frac{1}{2} \int_{\Omega}|\nabla \varphi(x)|^{2} \mathrm{~d} x & \text { if } \nabla \varphi \in L^{2}(\Omega), \\
+\infty & \text { otherwise, }\end{cases}
\end{gathered}
$$

whenever $\varphi_{\varepsilon} \rightarrow \varphi$ in $L^{2}(\Omega)$. Note that the limiting energy contribution on the righthand side is the potential associated to the negative Laplacian with homogeneous Neumann boundary conditions. Hence, this implicitly reveals that the "correct" choice of boundary condition arising for $u$ in the local limit is of Neumann type. Such idea is indeed proved rigorously performing the local asymptotics on the variational formulation for the nonlocal problem (1.1)-(1.4). The advantage of working using a variational approach is that the boundary conditions are implicitly contained in the variational formulation itself, and they have not to be tracked explicitly performing a pointwise analysis on the boundary.

We are now in a position to present the two main theorems that we prove in this paper. 
The first main result is the well-posedness for the nonlocal system (1.1)-(1.4) with Neumann boundary conditions when $\varepsilon>0$ is fixed. Here, the viscosity coefficient $\tau_{\varepsilon}$ is assumed to be strictly positive, the convolution kernel is of the form (1.11), and the double-well potential may be singular. In particular, we include in our analysis all the typical examples of polynomial, logarithmic, and double-obstacle potentials:

$$
\begin{aligned}
\Psi_{\text {pol }}(r) & :=\frac{1}{4}\left(r^{2}-1\right)^{2}, \quad r \in \mathbb{R}, \\
\Psi_{\text {log }}(r) & :=\frac{\vartheta}{2}[(1+r) \ln (1+r)+(1-r) \ln (1-r)]-\frac{\vartheta_{0}}{2}, \\
r \in(-1,1), \quad 0<\vartheta<\vartheta_{0}, & \\
\Psi_{\text {doub }}(r) & := \begin{cases}c\left(1-r^{2}\right) & \text { if } r \in[-1,1], \\
+\infty & \text { otherwise, }\end{cases}
\end{aligned}
$$

In view of this, the derivative of $\Psi$ is interpreted as a subdifferential in the sense of convex analysis, and equation (1.2) becomes a differential inclusion. The proof of well-posedness is based on a suitable approximation of the problem, given by a Yosida-type regularization on the nonlinearity and an additional elliptic local regularization in the chemical potential. A novel abstract variational setting $\left(V_{\varepsilon}, L^{2}(\Omega), V_{\varepsilon}^{*}\right)$ is introduced and uniform estimates on the approximated solutions are obtained. Using the viscous contribution in the chemical potential, strong compactness in $L^{2}$ is recovered even with no $H^{1}$-estimates on the solutions. Strong convergences are then proved and a passage to the limit provides solutions to the original nonlocal problem.

The second main result of this paper is the asymptotic analysis of the nonlocal system as $\varepsilon \searrow 0$. Here, we assume that the forcing terms $\left(g_{\varepsilon}\right)_{\varepsilon}$ converge to a certain source $g$, and that the viscosity coefficients satisfy

$$
\lim _{\varepsilon \searrow 0} \tau_{\varepsilon}=\tau
$$

Here, the coefficient $\tau$ is allowed to be nonnegative: when $\tau>0$ we obtain then nonlocal-to-local convergence of viscous Cahn-Hilliard equations, while if $\tau=$ 0 we obtain the local asymptotics of nonlocal viscous Cahn-Hilliard equations with vanishing viscosities. The proof is based on uniform estimates in $\varepsilon$ on the nonlocal solutions. Here, the strong compactness in $L^{2}$ is obtained by proving an ad-hoc compactness inequality involving the family on functional spaces $\left(V_{\varepsilon}\right)_{\varepsilon>0}$. The identification of the local limit $-\Delta u$ is obtained through the combination of monotone analysis techniques and Gamma-convergence results for the nonlocal energy functional (1.5).

We conclude by highlighting some possible applications of our results to phasefield modelling. The relevance of nonlocal-to-local convergence of Cahn-Hilliard equations with Neumann boundary conditions is significant: among many others, we can mention here possible connections with optimal control of tumor growth models. In the recent years, phase-field models have been widely used in tumor growth dynamics, both in the local case (see [25,31-35] and the references therein) 
and in the nonlocal case (see [26] and [48,54] for nonlocal Cahn-Hilliard equations with reaction terms). One of the main advantages of the nonlocal setting is that regularity results on the solutions are usually easier to obtain, not needing to rely on elliptic regularity properties. As a consequence, the availability of rigorous nonlocal-to-local convergence results would give the opportunity to approximate solutions to local phase-field systems with the solutions to the corresponding nonlocal ones, which are indeed simpler to handle on the mathematical side. For example, refined regularity on the solutions are fundamental when dealing with optimal control problems, in order to write first-order conditions for optimality. Hence, possible outcomes of nonlocal-to-local asymptotics concern refined analysis of optimal control of phase-field systems, in terms of passing to the (local) limit within first-order conditions for optimality for the nonlocal system.

The paper is structured in the following way: in Sect. 2 we state the assumptions, and we introduce the abstract variational settings. Section 3 is devoted to present the two main results. Section 4 contains the proof of well-posedness of the nonlocal system (1.1)-(1.4), while Sect. 5 focuses on the proof of nonlocal-to-local asymptotics.

\section{Mathematical Setting}

\subsection{Assumptions}

Throughout the paper, $\Omega$ is a smooth bounded domain in $\mathbb{R}^{d}$, with $d=2,3$, and $T>0$ is a fixed final time. We will use the notation $Q_{t}:=(0, t) \times \Omega$ for every $t \in(0, T]$, and set $Q:=Q_{T}$, and $\Sigma:=(0, T) \times \partial \Omega$. Moreover, $\left(\rho_{\varepsilon}\right)_{\varepsilon>0}$ is a family of mollifiers with the following properties (see [51,52]):

$$
\begin{aligned}
& \rho_{\varepsilon}: \mathbb{R} \rightarrow[0,+\infty), \quad \rho_{\varepsilon} \in L_{l o c}^{1}(\mathbb{R}), \quad \rho_{\varepsilon}(r)=\rho_{\varepsilon}(-r) \quad \forall r \in \mathbb{R}, \quad \forall \varepsilon>0 ; \\
& \int_{0}^{+\infty} \rho_{\varepsilon}(r) r^{d-1} \mathrm{~d} r=\frac{2}{C_{d}} \quad \forall \varepsilon>0 ; \\
& \lim _{\varepsilon \searrow 0} \int_{\delta}^{+\infty} \rho_{\varepsilon}(r) r^{d-1} \mathrm{~d} r=0 \quad \forall \delta>0,
\end{aligned}
$$

where $C_{d}:=\int_{S^{d-1}}\left|e_{1} \cdot \sigma\right|^{2} \mathrm{~d} \mathcal{H}^{d-1}(\sigma)$. We define the family of convolution kernels as

$$
\begin{aligned}
& K_{\varepsilon}: \Omega \times \Omega \rightarrow[0,+\infty), \quad K_{\varepsilon}(x, y):=\frac{\rho_{\varepsilon}(|x-y|)}{|x-y|^{2}}, \\
& \quad \text { for a.e. } x, y \in \Omega, \varepsilon>0 .
\end{aligned}
$$

Throughout the paper, $\gamma: \mathbb{R} \rightarrow 2^{\mathbb{R}}$ is a maximal monotone graph with $0 \in \gamma(0)$ and

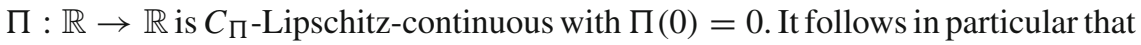
there exists a proper, convex, lower semicontinuous function $\hat{\gamma}: \mathbb{R} \rightarrow[0,+\infty]$ with $\hat{\gamma}(0)=0$ and $\partial \hat{\gamma}=\gamma$ in the sense of convex analysis. Similarly, we set $\hat{\Pi}(s):=\int_{0}^{s} \Pi(r) \mathrm{d} r$ for every $s \in \mathbb{R}$. With these notations, the double-well potential $\Psi$ entering the system is represented by the sum $\hat{\gamma}+\hat{\Pi}$. 


\subsection{Variational Setting and Preliminaries}

We introduce the functional spaces

$H:=L^{2}(\Omega), \quad V:=H^{1}(\Omega), \quad W:=\left\{\varphi \in H^{2}(\Omega): \partial_{\mathbf{n}} \varphi=0\right.$ a.e. on $\left.\partial \Omega\right\}$, endowed with their natural norms, and we identify $H$ with its dual space in the usual way, so that

$$
W \hookrightarrow V \hookrightarrow H \hookrightarrow V^{*} \hookrightarrow W^{*}
$$

where all the inclusions are continuous, dense, and compact. The Laplace operator with homogeneous Neumann conditions will be intended both as a bounded linear operator

$$
-\Delta: V \rightarrow V^{*}, \quad\langle-\Delta \varphi, \zeta\rangle_{V}:=\int_{\Omega} \nabla \varphi(x) \cdot \nabla \zeta(x) \mathrm{d} x, \quad \varphi, \zeta \in V,
$$

and as unbounded linear operator on $H$ with domain $W$. For every $\varphi \in V^{*}$, we use the notation $\varphi_{\Omega}:=\frac{1}{|\Omega|}\langle\varphi, 1\rangle_{V}$ for the mean value on $\Omega$. As a direct consequence of the Poincaré-Wirtinger inequality it holds that

$$
-\Delta:\left\{\varphi \in V: \varphi_{\Omega}=0\right\} \rightarrow\left\{\varphi \in V^{*}: \varphi_{\Omega}=0\right\}
$$

is a linear isomorphism. We will denote its inverse by

$$
\mathcal{N}:\left\{\varphi \in V^{*}: \varphi_{\Omega}=0\right\} \rightarrow\left\{\varphi \in V: \varphi_{\Omega}=0\right\} .
$$

For every $\varepsilon>0$, we set

$$
\begin{aligned}
V_{\varepsilon} & :=\left\{\varphi \in L^{2}(\Omega): \int_{\Omega} \int_{\Omega} K_{\varepsilon}(x, y)|\varphi(x)-\varphi(y)|^{2} \mathrm{~d} x \mathrm{~d} y<+\infty\right\}, \\
E_{\varepsilon}(\varphi) & :=\frac{1}{4} \int_{\Omega} \int_{\Omega} K_{\varepsilon}(x, y)|\varphi(x)-\varphi(y)|^{2} \mathrm{~d} x \mathrm{~d} y, \quad \varphi \in V_{\varepsilon} .
\end{aligned}
$$

Denoting by $a_{\varepsilon}: V_{\varepsilon} \times V_{\varepsilon} \rightarrow \mathbb{R}$ the natural bilinear form associated to $E_{\varepsilon}$, $a_{\varepsilon}(\varphi, \psi):=\frac{1}{2} \int_{\Omega} \int_{\Omega} K_{\varepsilon}(x, y)(\varphi(x)-\varphi(y))(\psi(x)-\psi(y)) \mathrm{d} x \mathrm{~d} y, \quad \varphi, \psi \in V_{\varepsilon}$, we also define

$$
\begin{aligned}
& W_{\varepsilon}:=\left\{\varphi \in V_{\varepsilon}: \exists f \in L^{2}(\Omega): a_{\varepsilon}(\varphi, \psi)=\int_{\Omega} f(x) \psi(x) \mathrm{d} x \text { for all } \psi \in V_{\varepsilon}\right\}, \\
& B_{\varepsilon}(\varphi)(x):=\int_{\Omega} K_{\varepsilon}(x, y)(\varphi(x)-\varphi(y)) \mathrm{d} y, \quad \text { for a.e. } x \in \Omega, \quad \varphi \in W_{\varepsilon} .
\end{aligned}
$$

Let us note that with such definitions the symmetry of $K_{\varepsilon}$ yields

$$
\begin{aligned}
& \left(B_{\varepsilon}(\varphi), \psi\right)_{H}=\frac{1}{2} \int_{\Omega} \int_{\Omega} K_{\varepsilon}(x, y)(\varphi(x) \\
& -\varphi(y))(\psi(x)-\psi(y)) \mathrm{d} x \mathrm{~d} y \\
& =a_{\varepsilon}(\varphi, \psi) \quad \forall \varphi, \psi \in W_{\varepsilon} .
\end{aligned}
$$


We point out that $E_{\varepsilon}: V_{\varepsilon} \rightarrow[0,+\infty)$ is convex and $B_{\varepsilon}: H \rightarrow H$ is a linear unbounded operator with domain $W_{\varepsilon}$. Additionally, we define the maps

$$
\|\cdot\|_{V_{\varepsilon}}: V_{\varepsilon} \rightarrow[0,+\infty), \quad\|\cdot\|_{W_{\varepsilon}}: W_{\varepsilon} \rightarrow[0,+\infty)
$$

as

$$
\|\varphi\|_{V_{\varepsilon}}:=\sqrt{\|\varphi\|_{H}^{2}+2 E_{\varepsilon}(\varphi)}, \quad\|\varphi\|_{W_{\varepsilon}}:=\sqrt{\|\varphi\|_{H}^{2}+\left\|B_{\varepsilon}(\varphi)\right\|_{H}^{2}},
$$

and the bilinear forms

$$
(\cdot, \cdot)_{V_{\varepsilon}}: V_{\varepsilon} \times V_{\varepsilon} \rightarrow[0,+\infty), \quad(\cdot, \cdot)_{W_{\varepsilon}}: W_{\varepsilon} \times W_{\varepsilon} \rightarrow[0,+\infty)
$$

as

$$
\begin{aligned}
\left(\varphi_{1}, \varphi_{2}\right)_{V_{\varepsilon}}: & =\left(\varphi_{1}, \varphi_{2}\right)_{H} \\
& +\frac{1}{2} \int_{\Omega} \int_{\Omega} K_{\varepsilon}(x, y)\left(\varphi_{1}(x)-\varphi_{1}(y)\right)\left(\varphi_{2}(x)-\varphi_{2}(y)\right) \mathrm{d} x \mathrm{~d} y, \\
\left(\varphi_{1}, \varphi_{2}\right)_{W_{\varepsilon}}: & =\left(\varphi_{1}, \varphi_{2}\right)_{H}+\left(B_{\varepsilon}\left(\varphi_{1}\right), B_{\varepsilon}\left(\varphi_{2}\right)\right)_{H} .
\end{aligned}
$$

We collect some properties in the next lemma.

Lemma 1. The following properties hold for every $\varepsilon>0$ :

(1) The spaces $V_{\varepsilon}$ and $W_{\varepsilon}$, endowed with the norms $\|\cdot\|_{V_{\varepsilon}}$ and $\|\cdot\|_{W_{\varepsilon}}$, respectively, are complete.

(2) The bilinear forms $(\cdot, \cdot)_{V_{\varepsilon}}$ and $(\cdot, \cdot)_{W_{\varepsilon}}$ are scalar products on $V_{\varepsilon}$ and $W_{\varepsilon}$ inducing the norms $\|\cdot\|_{V_{\varepsilon}}$ and $\|\cdot\|_{W_{\varepsilon}}$, respectively. In particular, $V_{\varepsilon}$ and $W_{\varepsilon}$ are Hilbert spaces.

(3) For every $\sigma \in(0,1]$ we have $C^{0, \sigma}(\bar{\Omega}) \hookrightarrow W_{\varepsilon}$ continuously, and there exists $C_{\varepsilon, \sigma}>0$ such that

$$
B_{\varepsilon}(\varphi) \in L^{\infty}(\Omega), \quad\left\|B_{\varepsilon}(\varphi)\right\|_{L^{\infty}(\Omega)} \leqq C_{\varepsilon, \sigma}\|\varphi\|_{C^{0, \sigma}(\bar{\Omega})} \quad \forall \varphi \in C^{0, \sigma}(\bar{\Omega}) .
$$

(4) The following inclusions are continuous and dense:

$$
W_{\varepsilon} \hookrightarrow V_{\varepsilon} \hookrightarrow H \text {. }
$$

Moreover, $B_{\varepsilon}: \mathcal{D}\left(B_{\varepsilon}\right) \subset H \rightarrow H$, with $\mathcal{D}\left(B_{\varepsilon}\right)=W_{\varepsilon}$, is maximal monotone on $\mathrm{H}$.

(5) The unbounded linear operator $B_{\varepsilon}: H \rightarrow H$ extends to a bounded linear operator $B_{\varepsilon}: V_{\varepsilon} \rightarrow V_{\varepsilon}^{*}$, and it holds that

$$
\left\|B_{\varepsilon}(\varphi)\right\|_{V_{\varepsilon}^{*}} \leqq\|\varphi\|_{V_{\varepsilon}} \quad \forall \varphi \in V_{\varepsilon} .
$$

Moreover, such extension coincides with the linear operator $A_{\varepsilon}: V_{\varepsilon} \rightarrow V_{\varepsilon}^{*}$ associated to the bilinear form $a_{\varepsilon}$, defined as

$$
A_{\varepsilon}(\varphi):=a_{\varepsilon}(\varphi, \cdot), \quad \varphi \in V_{\varepsilon} .
$$

(6) The map $E_{\varepsilon}: V_{\varepsilon} \rightarrow[0,+\infty)$ is of class $C^{1}$ and $D E_{\varepsilon}=B_{\varepsilon}: V_{\varepsilon} \rightarrow V_{\varepsilon}^{*}$. 
Proof. STEP I: properties (1)-(2). It is clear that $\|\cdot\|_{V_{\varepsilon}}$ and $\|\cdot\|_{W_{\varepsilon}}$ are norms on $V_{\varepsilon}$ and $W_{\varepsilon}$, respectively. Let now $\left(y_{n}\right)_{n}$ be a Cauchy sequence in $V_{\varepsilon}$ : then in particular it is a Cauchy sequence in $H$, so there exists $y \in H$ such that $y_{n} \rightarrow y$ in $H$. By lower semicontinuity it follows that $y \in V_{\varepsilon}$ as well, and that $y_{n} \rightarrow y$ in $V_{\varepsilon}$. A similar argument shows that $W_{\varepsilon}$ is complete as well. A direct computation shows that $(\cdot, \cdot)_{V_{\varepsilon}}$ and $(\cdot, \cdot)_{W_{\varepsilon}}$ are scalar products inducing the norms above.

STEP 2: property (3). For every $\varphi \in C^{0, \sigma}(\bar{\Omega})$, we have

$$
\left|B_{\varepsilon}(\varphi(x))\right| \leqq \int_{\Omega} \rho_{\varepsilon}(|x-y|) \frac{|\varphi(x)-\varphi(y)|}{|x-y|^{2}} \mathrm{~d} y \leqq\|\varphi\|_{C^{0, \sigma}(\bar{\Omega})} \int_{\Omega} \frac{\rho_{\varepsilon}(|x-y|)}{|x-y|^{2-\sigma}} \mathrm{d} y,
$$

where

$$
\begin{aligned}
\int_{\Omega} \frac{\rho_{\varepsilon}(|x-y|)}{|x-y|^{2-\sigma}} \mathrm{d} y= & \int_{\Omega-x} \frac{\rho_{\varepsilon}(|z|)}{|z|^{2-\sigma}} \mathrm{d} z \leqq \int_{\mathbb{R}^{d}} \frac{\rho_{\varepsilon}(|z|)}{|z|^{2-\sigma}} \mathrm{d} z=\int_{\{|z| \leqq 1\}} \frac{\rho_{\varepsilon}(|z|)}{|z|^{2-\sigma}} \mathrm{d} z \\
& +\int_{\{|z|>1\}} \frac{\rho_{\varepsilon}(|z|)}{|z|^{2-\sigma}} \mathrm{d} z \\
& \leqq \max _{|r| \leqq 1} \rho_{\varepsilon}(r) \int_{\{|z| \leqq 1\}} \frac{1}{|z|^{2-\sigma}} \mathrm{d} z+\int_{\{|z|>1\}} \rho_{\varepsilon}(|z|) \mathrm{d} z .
\end{aligned}
$$

The first term on the right-hand side is finite since $2-\sigma<d$, while the second term can be written as

$$
\left|S^{d-1}\right| \int_{1}^{+\infty} \rho_{\varepsilon}(r) r^{d-1} \mathrm{~d} r<+\infty
$$

by the assumptions on $\left(\rho_{\varepsilon}\right)_{\varepsilon}$. The thesis follows by the arbitrariness of $x \in \Omega$.

STEP 3: property (4). First of all the fact that the inclusion $V_{\varepsilon} \hookrightarrow H$ is continuous is trivial by the definition of $\|\cdot\|_{V_{\varepsilon}}$. Second, for $\varphi \in W_{\varepsilon}$, a direct computation shows that

$$
\begin{aligned}
E_{\varepsilon}(\varphi) & =\frac{1}{4} \int_{\Omega} \int_{\Omega} K_{\varepsilon}(x, y)|\varphi(x)-\varphi(y)|^{2} \mathrm{~d} x \mathrm{~d} y \\
& =\frac{1}{2} \int_{\Omega} B_{\varepsilon}(\varphi(x)) \varphi(x) \mathrm{d} x \\
& \leqq \frac{1}{2}\left\|B_{\varepsilon}(\varphi)\right\|_{H}\|\varphi\|_{H},
\end{aligned}
$$

so that $W_{\varepsilon} \hookrightarrow V_{\varepsilon}$ continuously. The density of $V_{\varepsilon}$ in $H$ follows from the density of $C^{0, \sigma}(\bar{\Omega})$ in $H$ and the fact that $C^{0, \sigma}(\bar{\Omega}) \subset W_{\varepsilon} \subset V_{\varepsilon}$.

The monotonicity of $B_{\varepsilon}$ is a direct consequence of its definition. We proceed by showing that it is maximal monotone. Let $\varphi \in H$. For every $\lambda, \delta>0$ the elliptic problem

$$
\begin{cases}\varphi_{\delta \lambda}+\lambda \Delta^{2} \varphi_{\delta \lambda}+\delta B_{\varepsilon}\left(\varphi_{\delta \lambda}\right)=\varphi & \text { in } \Omega, \\ \partial_{\mathbf{n}} \varphi_{\delta \lambda}=\partial_{\mathbf{n}} \Delta \varphi_{\delta \lambda}=0 & \text { on } \partial \Omega\end{cases}
$$

admits a unique weak solution $\varphi_{\delta \lambda} \in W \hookrightarrow C^{0,1 / 4}(\bar{\Omega}) \hookrightarrow W_{\varepsilon}$, in the sense that

$$
\left(\varphi_{\delta \lambda}, \psi\right)_{H}+\lambda\left(\Delta \varphi_{\delta \lambda}, \Delta \psi\right)_{H}+\delta\left(B_{\varepsilon}\left(\varphi_{\delta \lambda}\right), \psi\right)_{H}=(\varphi, \psi)_{H} \quad \forall \psi \in W .
$$


Taking arbitrary $\psi \in C_{c}^{\infty}(\Omega)$ in this variational formulation, we infer that $\Delta^{2} \varphi_{\delta \lambda} \in H$ in the sense of distributions. Consequently, the classical elliptic regularity theory yields also that $\varphi_{\delta \lambda} \in H^{4}(\Omega)$, with $\partial_{\mathbf{n}} \Delta \varphi_{\delta \lambda}=0$ almost everywhere on $\partial \Omega$. Fix now $\delta>0$. Testing (2.2) by $\varphi_{\delta \lambda}$ and using the monotonicity of $B_{\varepsilon}$ and the Young inequality, it follows that

$$
\left\|\varphi_{\delta \lambda}\right\|_{H}^{2}+\lambda\left\|\Delta \varphi_{\delta \lambda}\right\|_{H}^{2}+2 \delta E_{\varepsilon}\left(\varphi_{\delta \lambda}\right) \leqq \frac{1}{2}\|\varphi\|_{H}^{2}+\frac{1}{2}\left\|\varphi_{\delta \lambda}\right\|_{H}^{2} \quad \forall \lambda>0 .
$$

Thus, there exists a positive constant $M$ such that we have

$$
\left\|\varphi_{\delta \lambda}\right\|_{V_{\varepsilon}}^{2}+\lambda\left\|\Delta \varphi_{\delta \lambda}\right\|_{H}^{2} \leqq M \quad \forall \lambda>0 .
$$

Noting that for all $\zeta \in V_{\varepsilon}$, by the symmetry of the kernel $K_{\varepsilon}$ and the Hölder inequality we have

$$
\begin{aligned}
\left(B_{\varepsilon}\left(\varphi_{\delta \lambda}\right), \zeta\right)_{H} & =\frac{1}{2} \int_{\Omega} \int_{\Omega} K_{\varepsilon}(x, y)\left(\varphi_{\delta \lambda}(x)-\varphi_{\delta \lambda}(y)\right)(\zeta(x)-\zeta(y)) \mathrm{d} x \mathrm{~d} y \\
& \leqq 2\left\|\varphi_{\delta \lambda}\right\|_{V_{\varepsilon}}\|\zeta\|_{V_{\varepsilon}}
\end{aligned}
$$

the estimates just proved ensure also that

$$
\left\|B_{\varepsilon}\left(\varphi_{\delta \lambda}\right)\right\|_{V_{\varepsilon}^{*}} \leqq M
$$

We infer that there exist $\varphi_{\delta} \in V_{\varepsilon}$ and $\eta_{\delta} \in V_{\varepsilon}^{*}$ such that, as $\lambda \searrow 0, \lambda \varphi_{\delta \lambda} \rightarrow 0$ in $W, \varphi_{\delta \lambda} \rightarrow \varphi_{\delta}$ in $V_{\varepsilon}$, and $B_{\varepsilon}\left(\varphi_{\delta \lambda}\right) \rightarrow \eta_{\delta}$ in $V_{\varepsilon}^{*}$. It follows that

$$
\left(\varphi_{\delta}, \zeta\right)_{H}+\delta\left\langle\eta_{\delta}, \zeta\right\rangle_{V_{\varepsilon}^{*}, V_{\varepsilon}}=(\varphi, \zeta)_{H} \quad \forall \zeta \in V_{\varepsilon}
$$

Now, for all $\zeta \in V_{\varepsilon}$, by the symmetry of $B_{\varepsilon}$ and the bilinearity of $a_{\varepsilon}$ if holds that

$$
\left\langle\eta_{\delta}, \zeta\right\rangle_{V_{\varepsilon}^{*}, V_{\varepsilon}}=\lim _{\lambda \rightarrow 0}\left(B_{\varepsilon}\left(\varphi_{\delta \lambda}\right), \zeta\right)_{H}=\lim _{\lambda \rightarrow 0} a_{\varepsilon}\left(\varphi_{\delta \lambda}, \zeta\right)=a_{\varepsilon}\left(\varphi_{\delta}, \zeta\right)
$$

This shows that

$$
\delta a_{\varepsilon}\left(\varphi_{\delta}, \zeta\right)=\left(\varphi-\varphi_{\delta}, \zeta\right)_{H} \quad \forall \zeta \in V_{\varepsilon},
$$

so we conclude that $\varphi_{\delta} \in W_{\varepsilon}$ and $\eta_{\delta}=B_{\varepsilon}\left(\varphi_{\delta}\right)$. Hence,

$$
\varphi_{\delta}+\delta B_{\varepsilon}\left(\varphi_{\delta}\right)=\varphi \quad \forall \delta>0 .
$$

This proves that $B_{\varepsilon}$ is a maximal monotone operator on $H$ (see [4, Thm. 2.2]). Testing now (2.3) by $\varphi_{\delta}$ and using Young inequality it is immediate to see that

$$
\frac{1}{2}\left\|\varphi_{\delta}\right\|_{H}^{2}+\delta a_{\varepsilon}\left(\varphi_{\delta}, \varphi_{\delta}\right) \leqq \frac{1}{2}\|\varphi\|_{H}^{2} .
$$


Let us note that since we have just proved that $\varphi_{\delta} \in W_{\varepsilon}$, in particular we have that $B_{\varepsilon}\left(\varphi_{\delta}\right) \in H$. Hence, if additionally $\varphi \in V_{\varepsilon}$, testing (2.3) by $B_{\varepsilon}\left(\varphi_{\delta}\right)$ and using Hölder and Young inequalities yields

$$
\begin{aligned}
2 E_{\varepsilon}\left(\varphi_{\delta}\right)+\delta\left\|B_{\varepsilon}\left(\varphi_{\delta}\right)\right\|_{H}^{2} & =a_{\varepsilon}\left(\varphi_{\delta}, \varphi_{\delta}\right)+\delta\left\|B_{\varepsilon}\left(\varphi_{\delta}\right)\right\|_{H}^{2}=\left(B_{\varepsilon}\left(\varphi_{\delta}\right), \varphi\right)_{H} \\
& =\frac{1}{2} \int_{\Omega} \int_{\Omega} K_{\varepsilon}(x, y)\left(\varphi_{\delta}(x)-\varphi_{\delta}(y)\right)(\varphi(x)-\varphi(y)) \mathrm{d} x \mathrm{~d} y \\
& \leqq 2 \sqrt{E_{\varepsilon}(\varphi)} \sqrt{E_{\varepsilon}\left(\varphi_{\delta}\right)} \leqq E_{\varepsilon}\left(\varphi_{\delta}\right)+E_{\varepsilon}(\varphi)
\end{aligned}
$$

We deduce that, as $\delta \searrow 0, \delta B_{\varepsilon}\left(\varphi_{\delta}\right) \rightarrow 0$ in $H$. Hence, by (2.3), $\varphi_{\delta} \rightarrow \varphi$ in $H$. By combining (2.4) and (2.5), we obtain that $\left\|\varphi_{\delta}\right\|_{V_{\varepsilon}} \leqq\|\varphi\|_{V_{\varepsilon}}$ for every $\delta>0$. As $V_{\varepsilon}$ is uniformly convex, this implies that $\varphi_{\delta} \rightarrow \varphi$ in $V_{\varepsilon}$, so that $W_{\varepsilon} \hookrightarrow V_{\varepsilon}$ densely.

STEP 4: property (5). For every $\varphi \in W_{\varepsilon}$ and $\zeta \in V_{\varepsilon}$, by the Hölder inequality we have

$$
\begin{aligned}
\left(B_{\varepsilon}(\varphi), \zeta\right)_{H} & =\frac{1}{2} \int_{\Omega} \int_{\Omega} K_{\varepsilon}(x, y)(\varphi(x)-\varphi(y))(\zeta(x)-\zeta(y)) \mathrm{d} x \mathrm{~d} y \\
& \leqq 2 \sqrt{E_{\varepsilon}(\varphi)} \sqrt{E_{\varepsilon}(\zeta)}
\end{aligned}
$$

This implies that for every $\varphi \in W_{\varepsilon}$, the operator

$$
\zeta \mapsto\left(B_{\varepsilon}(\varphi), \zeta\right)_{H}, \quad \zeta \in V_{\varepsilon},
$$

is linear and continuous on $V_{\varepsilon}$, and such that

$$
\left\|\zeta \mapsto\left(B_{\varepsilon}(\varphi), \zeta\right)_{H}\right\|_{V_{\varepsilon}^{*}} \leqq\|\varphi\|_{V_{\varepsilon}} \quad \forall \varphi \in W_{\varepsilon} .
$$

Since $W_{\varepsilon} \hookrightarrow V_{\varepsilon}$ is dense, we deduce that $B_{\varepsilon}$ extends to a bounded linear operator from $V_{\varepsilon}$ to $V_{\varepsilon}^{*}$, and the thesis follows.

STEP 5: property (6). We observe that $E_{\varepsilon}: V_{\varepsilon} \rightarrow[0,+\infty)$ is convex and lower semicontinuous. A direct computation also shows that $D E_{\varepsilon}=B_{\varepsilon}$ in the sense of Gâteaux: since $B_{\varepsilon}: V_{\varepsilon} \rightarrow V_{\varepsilon}^{*}$ is linear and continuous, the thesis follows.

The next lemma shows some boundedness properties of the family $\left(B_{\varepsilon}\right)_{\varepsilon}$, uniformly in $\varepsilon$.

Lemma 2. The following inclusion is continuous:

$$
V \hookrightarrow V_{\varepsilon},
$$

and there exists a constant $C$, independent of $\varepsilon$, such that

$$
\|\varphi\|_{V_{\varepsilon}} \leqq C\|\varphi\|_{V} \quad \forall \varphi \in V .
$$

For every $\varphi, \zeta \in V$, if holds that

$$
\lim _{\varepsilon \searrow 0} E_{\varepsilon}(\varphi)=\frac{1}{2} \int_{\Omega}|\nabla \varphi(x)|^{2} d x, \quad \lim _{\varepsilon \searrow 0}\left\langle B_{\varepsilon}\left(\varphi_{1}\right), \varphi_{2}\right\rangle_{V_{\varepsilon}}=\int_{\Omega} \nabla \varphi_{1}(x) \cdot \nabla \varphi_{2}(x) d x .
$$


Finally, for every $\varphi \in H$ and for every sequence $\left(\varphi_{\varepsilon}\right)_{\varepsilon>0} \subset H$ with $\varphi_{\varepsilon} \rightarrow \varphi$ in $H$, we have

$$
\liminf _{\varepsilon \searrow 0} E_{\varepsilon}\left(\varphi_{\varepsilon}\right) \geqq E(\varphi):= \begin{cases}\frac{1}{2} \int_{\Omega}|\nabla \varphi(x)|^{2} d x & \text { if } \varphi \in V, \\ +\infty & \text { if } \varphi \in H \backslash V .\end{cases}
$$

In other words, $\left(E_{\varepsilon}\right)_{\varepsilon>0} \Gamma$-converges to $E$ with respect to the norm-topology of $H$.

Proof. By [52], there is a constant $C>0$ independent of $\varepsilon$ such that

$$
E_{\varepsilon}(\varphi) \leqq C\|\nabla \varphi\|_{H}^{2} \quad \forall \varphi \in V
$$

from which the first part of the thesis follows directly. The first limit in (2.6) is also a direct consequence of [52], the second limit in (2.6) can be proved by choosing $\varphi=\varphi_{1} \pm \varphi_{2}$ in the first limit.

Finally, by the $\Gamma$-convergence result in $[51, \mathrm{Thm} .8]$, we know that

$$
\liminf _{\varepsilon \searrow 0} E_{\varepsilon}\left(\varphi_{\varepsilon}\right) \geqq \mathrm{sc}-\tilde{E}(\varphi),
$$

where sc $-\tilde{E}$ is the lower semicontinuous envelope of

$$
\tilde{E}: H \rightarrow[0,+\infty], \quad \tilde{E}(\varphi):= \begin{cases}\frac{1}{2} \int_{\Omega}|\nabla \varphi(x)|^{2} \mathrm{~d} x & \text { if } \varphi \in C^{1}(\bar{\Omega}), \\ +\infty & \text { otherwise }\end{cases}
$$

i.e.

$$
\mathrm{sc}-\tilde{E}(\varphi)=\inf \left\{\liminf _{n \rightarrow \infty} \tilde{E}\left(\zeta_{n}\right): \zeta_{n} \rightarrow \varphi \text { in } H\right\}
$$

It is a standard matter to check that $\mathrm{sc}-\tilde{E}=E$, so that the thesis follows.

The last result of this section is a compactness criterion involving the family of operators $\left(E_{\varepsilon}\right)_{\varepsilon}$. The following lemma is fundamental as we do not have any compactness properties for the inclusions of the spaces $V_{\varepsilon}$ and $W_{\varepsilon}$. For the proof we refer to [24, Lemma. 4].

Lemma 3. For every $\delta>0$ there exist two constants $C_{\delta}>0$ and $\varepsilon_{\delta}>0$ such that, for every sequence $\left(\varphi_{\varepsilon}\right)_{\varepsilon \in\left(0, \varepsilon_{\delta}\right)} \subset V_{\varepsilon}$ if holds that

$$
\left\|\varphi_{\varepsilon_{1}}-\varphi_{\varepsilon_{2}}\right\|_{H}^{2} \leqq \delta\left(E_{\varepsilon_{1}}\left(\varphi_{\varepsilon_{1}}\right)+E_{\varepsilon_{2}}\left(\varphi_{\varepsilon_{2}}\right)\right)+C_{\delta}\left\|\varphi_{\varepsilon_{1}}-\varphi_{\varepsilon_{2}}\right\|_{V^{*}}^{2} \forall \varepsilon_{1}, \varepsilon_{2} \in\left(0, \varepsilon_{\delta}\right) .
$$

\section{Main Results}

Before stating our main results, we recall that the local Cahn-Hilliard equation is well-posed in the following sense: 
Theorem 3.1. Let $\tau \geqq 0$ and

$$
\begin{aligned}
& u_{0} \in V, \quad \hat{\gamma}\left(u_{0}\right) \in L^{1}(\Omega), \quad\left(u_{0}\right)_{\Omega} \in \operatorname{Int} D(\gamma), \\
& g \in L^{2}(0, T ; H), \quad g \in H^{1}(0, T ; H) \quad \text { if } \tau=0 .
\end{aligned}
$$

Then, there exists a triple $(u, \mu, \xi)$ such that

$$
\begin{aligned}
& u \in H^{1}\left(0, T ; V^{*}\right) \cap L^{\infty}(0, T ; V) \cap L^{2}(0, T ; W), \quad \tau u \in H^{1}(0, T ; H), \\
& \mu \in L^{2}(0, T ; V), \quad \tau \mu \in L^{2}(0, T ; W), \\
& \xi \in L^{2}(0, T ; H), \quad \xi \in \gamma(u) \quad \text { a.e.in } Q, \\
& \partial_{t} u-\Delta \mu=0 \text { in } V^{*}, \quad \text { a.e. in }(0, T), \\
& \mu=\tau \partial_{t} u-\Delta u+\xi+\Pi(u)-g \text { a.e.in } Q, \\
& u(0)=u_{0} \quad \text { a.e.in } \Omega \text {. }
\end{aligned}
$$

Moreover, the solution component $u$ is unique, and the solution components $\mu$ and $\xi$ are unique if $\gamma$ is single-valued.

Proof. We refer to [18] for a proof in a more general setting.

The first result of this paper is the well-posedness of the nonlocal viscous CahnHilliard equation complemented by Neumann boundary conditions for the chemical potential.

Theorem 3.2. Let $\varepsilon>0$ and $\tau_{\varepsilon}>0$ be fixed. Then for every $\left(u_{0, \varepsilon}, g_{\varepsilon}\right)$ with

$$
\begin{aligned}
& u_{0, \varepsilon} \in V_{\varepsilon}, \quad \hat{\gamma}\left(u_{0, \varepsilon}\right) \in L^{1}(\Omega), \quad\left(u_{0, \varepsilon}\right)_{\Omega} \in \operatorname{Int} D(\gamma), \\
& g_{\varepsilon} \in L^{2}(0, T ; H),
\end{aligned}
$$

there exists a triple $\left(u_{\varepsilon}, \mu_{\varepsilon}, \xi_{\varepsilon}\right)$ such that

$$
\begin{aligned}
& u_{\varepsilon} \in H^{1}(0, T ; H) \cap L^{\infty}\left(0, T ; V_{\varepsilon}\right) \cap L^{2}\left(0, T ; W_{\varepsilon}\right), \\
& \mu_{\varepsilon} \in L^{2}(0, T ; W), \\
& \xi_{\varepsilon} \in L^{2}(0, T ; H), \quad \xi_{\varepsilon} \in \gamma\left(u_{\varepsilon}\right) \text { a.e. in } Q, \\
& \partial_{t} u_{\varepsilon}-\Delta \mu_{\varepsilon}=0 \text { a.e. in } Q, \\
& \mu_{\varepsilon}=\tau_{\varepsilon} \partial_{t} u_{\varepsilon}+B_{\varepsilon}\left(u_{\varepsilon}\right)+\xi_{\varepsilon}+\Pi\left(u_{\varepsilon}\right)-g_{\varepsilon} \text { a.e. in } Q, \\
& u_{\varepsilon}(0)=u_{0, \varepsilon} \text { a.e. in } \Omega .
\end{aligned}
$$

Furthermore, there exists a positive constant $M_{\varepsilon}$ such that, for every sets of data $\left(u_{0, \varepsilon}^{1}, g_{\varepsilon}^{1}\right)$ and $\left(u_{0, \varepsilon}^{2}, g_{\varepsilon}^{2}\right)$ satisfying (3.9)-(3.10), with $\left(u_{0, \varepsilon}^{1}\right)_{\Omega}=\left(u_{0, \varepsilon}^{2}\right)_{\Omega}$, and for every respective solutions $\left(u_{\varepsilon}^{1}, \mu_{\varepsilon}^{1}, \xi_{\varepsilon}^{1}\right)$ and $\left(u_{\varepsilon}^{2}, \mu_{\varepsilon}^{2}, \xi_{\varepsilon}^{2}\right)$ satisfying (3.11)-(3.16), it holds

$$
\begin{aligned}
& \left\|u_{\varepsilon}^{1}-u_{\varepsilon}^{2}\right\|_{C^{0}\left([0, T] ; V^{*}\right)}^{2}+\tau_{\varepsilon}\left\|u_{\varepsilon}^{1}-u_{\varepsilon}^{2}\right\|_{C^{0}([0, T] ; H)}^{2}+\left\|E_{\varepsilon}\left(u_{\varepsilon}^{1}-u_{\varepsilon}^{2}\right)\right\|_{L^{1}(0, T)} \\
& \quad \leqq M_{\varepsilon}\left(\left\|u_{0, \varepsilon}^{1}-u_{0, \varepsilon}^{2}\right\|_{V^{*}}^{2}+\tau_{\varepsilon}\left\|u_{0, \varepsilon}^{1}-u_{0, \varepsilon}^{2}\right\|_{H}^{2}+\left\|g_{\varepsilon}^{1}-g_{\varepsilon}^{2}\right\|_{L^{2}\left(0, T ; V^{*}\right)}^{2}\right) .
\end{aligned}
$$

In particular, the solution component $u_{\varepsilon}$ is unique, and the solution components $\mu_{\varepsilon}$ and $\xi_{\varepsilon}$ are unique if $\gamma$ is single-valued. 
Our second contribution concerns the nonlocal-to-local convergence. In particular, we show that, under suitable assumptions on the initial data $\left(u_{0, \varepsilon}\right)_{\varepsilon}$ and on the forcing terms $\left(g_{\varepsilon}\right)_{\varepsilon}$, if the viscosities $\left(\tau_{\varepsilon}\right)_{\varepsilon}$ converge to a coefficient $\tau \geqq 0$, then the solutions to the respective viscous nonlocal Cahn-Hilliard equations converge, in suitable topologies, to the solutions to the limiting local Cahn-Hilliard equation with viscosity parameter $\tau \geqq 0$. Note that the viscosities $\left(\tau_{\varepsilon}\right)_{\varepsilon}$ are required to be strictly positive for all $\varepsilon>0$, whereas the limiting viscosity parameter $\tau$ is also allowed to vanish. Hence, such result has a duplex formulation. Indeed, if $\tau>0$ this shows the asymptotic convergence of the nonlocal viscous equation to the corresponding local viscous equation, while if $\tau=0$ this proves the approximability of solutions to the local pure equation by solutions to nonlocal equations with vanishing viscosities.

Theorem 3.3. Assume that

$$
\tau \geqq 0, \quad\left(\tau_{\varepsilon}\right)_{\varepsilon>0} \subset(0,+\infty), \quad \lim _{\varepsilon \searrow 0} \tau_{\varepsilon}=\tau .
$$

Let the data $\left(u_{0}, g\right)$ satisfy (3.1)-(3.2), and let the family $\left(u_{0, \varepsilon}, g_{\varepsilon}\right)_{\varepsilon>0}$ satisfy (3.9)(3.10) for all $\varepsilon>0$. Assume also that there exists $\varepsilon_{0}>0$ such that

$$
\begin{aligned}
& \sup _{\varepsilon \in\left(0, \varepsilon_{0}\right)}\left(\left\|u_{0, \varepsilon}\right\|_{V_{\varepsilon}}^{2}+\left\|\hat{\gamma}\left(u_{0, \varepsilon}\right)\right\|_{L^{1}(\Omega)}\right)<+\infty \\
& \left(g_{\varepsilon}\right)_{\varepsilon \in\left(0, \varepsilon_{0}\right)} \subset H^{1}(0, T ; H) \text { and } \sup _{\varepsilon \in\left(0, \varepsilon_{0}\right)}\left\|g_{\varepsilon}\right\|_{H^{1}(0, T ; H)}^{2}<+\infty \text { if } \tau=0 \\
& \exists\left[a_{0}, b_{0}\right] \subset \text { Int } D(\gamma): \quad a_{0} \leqq\left(u_{0, \varepsilon}\right)_{\Omega} \leqq b_{0} \quad \forall \varepsilon \in\left(0, \varepsilon_{0}\right) \\
& u_{0, \varepsilon} \rightarrow u_{0} \text { in } H \text { as } \varepsilon \searrow 0, \quad g_{\varepsilon} \rightarrow g \text { in } L^{2}(0, T ; H) \text { as } \varepsilon \searrow 0
\end{aligned}
$$

Let $\left(u_{\varepsilon}, \mu_{\varepsilon}, \xi_{\varepsilon}\right)_{\varepsilon \in\left(0, \varepsilon_{0}\right)}$ be a family of solutions to (3.11)-(3.16) corresponding to the data $\left(u_{0, \varepsilon}, g_{\varepsilon}\right)$ and viscosity $\tau_{\varepsilon}$, where $u_{\varepsilon}$ is uniquely determined. Then, there exists a solution $(u, \mu, \xi)$ to (3.3)-(3.8) corresponding to the data $\left(u_{0}, g\right)$ and viscosity $\tau$, where $u$ is uniquely determined, such that, as $\varepsilon \searrow 0$,

$$
\begin{aligned}
u_{\varepsilon} \rightarrow u & \text { in } C^{0}([0, T] ; H), \\
\partial_{t} u_{\varepsilon} \rightarrow \partial_{t} u & \text { in } L^{2}\left(0, T ; V^{*}\right), \\
\partial_{t} u_{\varepsilon} \rightarrow \partial_{t} u & \text { in } L^{2}(0, T ; H) \quad \text { if } \tau>0, \\
\tau_{\varepsilon} \partial_{t} u_{\varepsilon} \rightarrow 0 & \text { in } L^{2}(0, T ; H) \text { if } \tau=0, \\
\mu_{\varepsilon} \rightarrow \mu & \text { in } L^{2}(0, T ; V), \\
\mu_{\varepsilon} \rightarrow \mu & \text { in } L^{2}(0, T ; W) \text { if } \tau>0, \\
\xi_{\varepsilon} \rightarrow \xi & \text { in } L^{2}(0, T ; H) .
\end{aligned}
$$

\section{Proof of Theorem 3.2}

This section is devoted to the proof of well-posedness of the nonlocal viscous Cahn-Hilliard equation. Throughout the section, $\varepsilon>0$ and $\tau_{\varepsilon}>0$ are fixed. 


\subsection{Approximation}

For every $\lambda>0$, let $\gamma_{\lambda}: \mathbb{R} \rightarrow \mathbb{R}$ be the Yosida approximation of $\gamma$, having Lipschitz constant $1 / \lambda$, and set $\hat{\gamma}_{\lambda}(s):=\int_{0}^{s} \gamma_{\lambda}(r) \mathrm{d} r$ for every $s \in \mathbb{R}$. We consider the approximated problem

$$
\begin{aligned}
\partial_{t} u_{\varepsilon}^{\lambda}-\Delta \mu_{\varepsilon}^{\lambda}=0 & \text { in } Q, \\
\mu_{\varepsilon}^{\lambda}=\tau_{\varepsilon} \partial_{t} u_{\varepsilon}^{\lambda}-\lambda \Delta u_{\varepsilon}^{\lambda}+B_{\varepsilon}\left(u_{\varepsilon}^{\lambda}\right)+\gamma_{\lambda}\left(u_{\varepsilon}^{\lambda}\right)+\Pi\left(u_{\varepsilon}^{\lambda}\right)-g_{\varepsilon} & \text { in } Q, \\
\partial_{\mathbf{n}} u_{\varepsilon}^{\lambda}=\partial_{\mathbf{n}} \mu_{\varepsilon}^{\lambda}=0 & \text { in } \Sigma, \\
u_{\varepsilon}^{\lambda}(0)=u_{0, \varepsilon}^{\lambda} & \text { in } \Omega,
\end{aligned}
$$

where the initial datum $u_{0, \varepsilon}^{\lambda}$ satisfies

$$
\begin{aligned}
& u_{0, \varepsilon}^{\lambda} \in V, \quad u_{0, \varepsilon}^{\lambda} \rightarrow u_{0, \varepsilon} \text { in } H \text { as } \varepsilon \searrow 0, \\
& \sup _{\lambda \in\left(0, \lambda_{0}\right)}\left(\lambda\left\|u_{0, \varepsilon}^{\lambda}\right\|_{V}^{2}+\left\|\hat{\gamma}\left(u_{0, \varepsilon}^{\lambda}\right)\right\|_{L^{1}(\Omega)}\right)<+\infty
\end{aligned}
$$

for a certain $\lambda_{0}>0$ (possibly depending on $\varepsilon$ ). The existence of an approximating sequence $\left(u_{0, \varepsilon}^{\lambda}\right) \lambda$ satisfying (4.5)-(4.6) is guaranteed by assumption (3.1): for example, one can check that the classical elliptic regularization given by the unique solution to the problem

$$
\begin{cases}u_{0, \varepsilon}^{\lambda}-\lambda \Delta u_{0, \varepsilon}^{\lambda}=u_{0, \varepsilon} & \text { in } \Omega, \\ \partial_{\mathbf{n}} u_{0, \varepsilon}^{\lambda}=0 & \text { in } \partial \Omega,\end{cases}
$$

is a possible choice. The existence of a unique approximated solution $\left(u_{\varepsilon}^{\lambda}, \mu_{\varepsilon}^{\lambda}\right)$ for every $\lambda>0$ relies on a fixed-point argument, as in [24, Section 3.1]. For every $v \in L^{2}(0, T ; W)$, since $W \hookrightarrow C^{0, \frac{1}{4}}(\bar{\Omega})$ by the Sobolev embeddings, thanks to the properties of $B_{\varepsilon}$ proved in Lemma 1 we have that $B_{\varepsilon}(v) \in L^{2}(0, T ; H)$. Hence, by the classical literature on the local viscous Cahn-Hilliard equation (see again [18]), the map

$$
\begin{aligned}
& \Gamma_{\varepsilon}^{\lambda}: C^{0}([0, T] ; H) \cap L^{2}(0, T ; W) \rightarrow H^{1}(0, T ; H) \cap L^{\infty}(0, T ; V) \\
& \quad \cap L^{2}(0, T ; W), \Gamma_{\varepsilon}^{\lambda}: v \mapsto v_{\varepsilon}^{\lambda},
\end{aligned}
$$

is well-defined, where $\left(v_{\varepsilon}^{\lambda}, w_{\varepsilon}^{\lambda}\right)$ is the unique solution to the local viscous CahnHilliard equation

$$
\begin{aligned}
\partial_{t} v_{\varepsilon}^{\lambda}-\Delta w_{\varepsilon}^{\lambda}=0 & \text { in } Q, \\
w_{\varepsilon}^{\lambda}=\tau_{\varepsilon} \partial_{t} v_{\varepsilon}^{\lambda}-\lambda \Delta v_{\varepsilon}^{\lambda}+\gamma_{\lambda}\left(v_{\varepsilon}^{\lambda}\right)+\Pi\left(v_{\varepsilon}^{\lambda}\right)-\left(g_{\varepsilon}-B_{\varepsilon}(v)\right) & \text { in } Q, \\
\partial_{\mathbf{n}} u_{\varepsilon}^{\lambda}=\partial_{\mathbf{n}} \mu_{\varepsilon}^{\lambda}=0 & \text { in } \Sigma, \\
v_{\varepsilon}^{\lambda}(0)=u_{0, \varepsilon}^{\lambda} & \text { in } \Omega .
\end{aligned}
$$

Now, arguing as in [24, Section 3.1], exploiting the Lipschitz-continuity of $\gamma_{\lambda}$, the Sobolev embeddings, and the properties of $B_{\varepsilon}$ contained in Lemma 1, we 
deduce that there exist constants $L_{\varepsilon}^{\lambda}>0$ and $\sigma>0$ such that, for every $v_{1}, v_{2} \in$ $C^{0}([0, T] ; H) \cap L^{2}(0, T ; W)$, we have

$$
\left\|\Gamma_{\varepsilon}^{\lambda}\left(v_{1}\right)-\Gamma_{\varepsilon}^{\lambda}\left(v_{2}\right)\right\|_{C^{0}([0, T] ; H) \cap L^{2}(0, T ; W)} \leqq L_{\varepsilon}^{\lambda} T^{\sigma}\left\|v_{1}-v_{2}\right\|_{L^{2}(0, T ; W)} .
$$

It follows that one can choose $T_{0} \in(0, T]$ sufficiently small so that $\Gamma_{\varepsilon}^{\lambda}$ is a contraction on the respective functional spaces defined in $\left(0, T_{0}\right)$. Performing then a classical patching argument (we refer again to [24, Section 3.1] for details), we infer that $\Gamma_{\varepsilon}^{\lambda}$ has a unique fixed point on the whole interval $[0, T]$. This proves that the approximated system (4.1)-(4.4) has a unique solution

$$
u_{\varepsilon}^{\lambda} \in H^{1}(0, T ; H) \cap L^{\infty}(0, T ; V) \cap L^{2}(0, T ; W), \quad \mu_{\varepsilon}^{\lambda} \in L^{2}(0, T ; W) .
$$

\subsection{Uniform Estimates}

We prove here some uniform estimates independently of $\lambda$ and $\varepsilon$. In what follows we will always assume that $\lambda \in[0,1]$. Moreover, $\varepsilon>0$ and $\tau_{\varepsilon}>0$ are still fixed.

We start by fixing $t \in[0, T]$, testing (4.1) with $\mu_{\varepsilon}^{\lambda}$, (4.2) with $\partial_{t} u_{\varepsilon}^{\lambda}$, taking the difference, and integrating the resulting equation on $(0, t)$. We obtain

$$
\begin{aligned}
\int_{Q_{t}} & \left|\nabla \mu_{\varepsilon}^{\lambda}(s, x)\right|^{2} \mathrm{~d} x \mathrm{~d} s+\tau_{\varepsilon} \int_{Q_{t}}\left|\partial_{t} u_{\varepsilon}^{\lambda}(s, x)\right|^{2} \mathrm{~d} x \mathrm{~d} s \\
& +\frac{\lambda}{2} \int_{\Omega}\left|\nabla u_{\varepsilon}^{\lambda}(t, x)\right|^{2} \mathrm{~d} x+E_{\varepsilon}\left(u_{\varepsilon}^{\lambda}(t, \cdot)\right)+\int_{\Omega}\left(\hat{\gamma}_{\lambda}+\hat{\Pi}\right)\left(u_{\varepsilon}^{\lambda}(t, x)\right) \mathrm{d} x \\
\leqq & \frac{\lambda}{2} \int_{\Omega}\left|\nabla u_{0, \varepsilon}^{\lambda}(x)\right|^{2} \mathrm{~d} x+E_{\varepsilon}\left(u_{0, \varepsilon}^{\lambda}\right)+\int_{\Omega}\left(\hat{\gamma}_{\lambda}+\hat{\Pi}\right)\left(u_{0, \varepsilon}^{\lambda}(x)\right) \mathrm{d} x \\
& +\int_{Q_{t}}\left|g_{\varepsilon}(s, x)\right|\left|\partial_{t} u(s, x)\right| \mathrm{d} x \mathrm{~d} s .
\end{aligned}
$$

From the fact that

$$
\int_{\Omega} \hat{\gamma}_{\lambda}\left(u_{0, \varepsilon}^{\lambda}(x)\right) \mathrm{d} x \leqq \int_{\Omega} \hat{\gamma}\left(u_{0, \varepsilon}^{\lambda}(x)\right) \mathrm{d} x \text { for every } \lambda>0,
$$

using the uniform bound (4.6) as well as the Young inequality, we get

$$
\begin{aligned}
& \int_{Q_{t}}\left|\nabla \mu_{\varepsilon}^{\lambda}(s, x)\right|^{2} \mathrm{~d} x \mathrm{~d} s+\frac{\tau_{\varepsilon}}{2} \int_{Q_{t}}\left|\partial_{t} u_{\varepsilon}^{\lambda}(s, x)\right|^{2} \mathrm{~d} x \mathrm{~d} s \\
& \quad+E_{\varepsilon}\left(u_{\varepsilon}^{\lambda}(t, \cdot)\right)+\frac{\lambda}{2} \int_{\Omega}\left|\nabla u_{\varepsilon}^{\lambda}(t, x)\right|^{2} \mathrm{~d} x \\
& \leqq C_{\varepsilon}+\frac{\tau_{\varepsilon}}{4} \int_{Q_{t}}\left|\partial_{t} u_{\varepsilon}^{\lambda}(t, x)\right|^{2} \mathrm{~d} x \mathrm{~d} t+\frac{1}{\tau_{\varepsilon}} \int_{0}^{T} \int_{\Omega}\left|g_{\varepsilon}(t, x)\right|^{2} \mathrm{~d} x \mathrm{~d} t
\end{aligned}
$$

for every $t \in[0, T]$, where $C_{\varepsilon}>0$ is a constant independent of $\lambda$ and depending only on the initial datum $u_{0, \varepsilon}$.

From the arbitrariness of $t \in[0, T]$ we deduce that, for every $\lambda \in(0,1)$,

$$
\begin{aligned}
& \left\|\nabla \mu_{\varepsilon}^{\lambda}\right\|_{L^{2}(0, T ; H)} \leqq C_{\varepsilon}, \\
& \left\|u_{\varepsilon}^{\lambda}\right\|_{L^{\infty}\left(0, T ; V_{\varepsilon}\right)}+\left\|u_{\varepsilon}^{\lambda}\right\|_{H^{1}(0, T ; H)}+\lambda^{1 / 2}\left\|\nabla u_{\varepsilon}^{\lambda}\right\|_{L^{\infty}(0, T ; H)} \leqq C_{\varepsilon},
\end{aligned}
$$


hence, in addition by comparison in (4.1),

$$
\left\|\Delta \mu_{\varepsilon}^{\lambda}\right\|_{L^{2}(0, T ; H)} \leqq C_{\varepsilon} .
$$

Furthermore, noting that $\left(u_{\varepsilon}^{\lambda}\right)_{\Omega}=\left(u_{0, \varepsilon}^{\lambda}\right)_{\Omega}=\left(u_{0, \varepsilon}\right)_{\Omega}$, we test (4.1) by $\mathcal{N}\left(u_{\varepsilon}^{\lambda}-\right.$ $\left.\left(u_{0, \varepsilon}\right)_{\Omega}\right)$, (4.2) by $u_{\varepsilon}^{\lambda}-\left(u_{0, \varepsilon}\right)_{\Omega}$, and sum: we obtain, for almost every $t \in(0, T)$,

$$
\begin{aligned}
& \left\langle\partial_{t} u_{\varepsilon}^{\lambda}(t), \mathcal{N}\left(u_{\varepsilon}^{\lambda}(t)-\left(u_{0, \varepsilon}\right)_{\Omega}\right)\right\rangle_{V}+\tau_{\varepsilon}\left\langle\partial_{t} u_{\varepsilon}^{\lambda}(t), u_{\varepsilon}^{\lambda}(t)-\left(u_{0, \varepsilon}\right)_{\Omega}\right\rangle_{V} \\
& \quad+\lambda \int_{\Omega}\left|\nabla u_{\varepsilon}^{\lambda}(t, x)\right|^{2} \mathrm{~d} x \\
& \quad+\int_{\Omega} B_{\varepsilon}\left(u_{\varepsilon}^{\lambda}\right)(t, x) u_{\varepsilon}^{\lambda}(t, x) \mathrm{d} x+\int_{\Omega} \gamma_{\lambda}\left(u_{\lambda}(t, x)\right)\left(u_{\varepsilon}^{\lambda}(t, x)-\left(u_{0, \varepsilon}\right)_{\Omega}\right) \mathrm{d} x \\
& =\int_{\Omega}\left(g^{\varepsilon}(t, x)-\Pi\left(u_{\varepsilon}^{\lambda}\right)(t, x)\right)\left(u_{\varepsilon}^{\lambda}(t, x)-\left(u_{0, \varepsilon}\right)_{\Omega}\right) \mathrm{d} x,
\end{aligned}
$$

where we have used that $\int_{\Omega} B_{\varepsilon}\left(u_{\varepsilon}^{\lambda}(t, x)\right) \mathrm{d} x=0$ by the symmetry of the kernel $K_{\varepsilon}$. A classical argument shows that since $\left(u_{0, \varepsilon}\right)_{\Omega} \in \operatorname{Int} D(\gamma)$, then there are two constants $c_{\varepsilon}, c_{\varepsilon}^{\prime}$, only depending on the position of $\left(u_{0, \varepsilon}\right)_{\Omega}$, such that

$$
\begin{aligned}
& \left\|\gamma_{\lambda}\left(u_{\varepsilon}^{\lambda}(t, \cdot)\right)\right\|_{L^{1}(\Omega)} \leqq c_{\varepsilon} \int_{\Omega} \gamma_{\lambda}\left(u_{\varepsilon}^{\lambda}(t, x)\right)\left(u_{\varepsilon}^{\lambda}(t, x)-\left(u_{0, \varepsilon}\right)_{\Omega}\right) \mathrm{d} x+c_{\varepsilon}^{\prime}, \\
& \quad \text { for a.e. } t \in(0, T)
\end{aligned}
$$

Arguing as in [24, Subsection 3.2], the estimates above and (4.8)-(4.9) yield then a control on $\left\|\gamma_{\lambda}\left(u_{\lambda}\right)\right\|_{L^{2}\left(0, T ; L^{1}(\Omega)\right)}$. In particular, by comparison in (4.2) we get an estimate on $\left(\mu_{\varepsilon}^{\lambda}\right)_{\Omega}$ in $L^{2}(0, T)$. Taking (4.8) and (4.10) into account, we deduce then that

$$
\left\|\mu_{\varepsilon}^{\lambda}\right\|_{L^{2}(0, T ; W)} \leqq C_{\varepsilon}
$$

By comparison in (4.2) we infer that

$$
\left\|-\lambda \Delta u_{\varepsilon}^{\lambda}+B_{\varepsilon}\left(u_{\varepsilon}^{\lambda}\right)+\gamma_{\lambda}\left(u_{\varepsilon}^{\lambda}\right)\right\|_{L^{2}(0, T ; H)} \leqq C_{\varepsilon} .
$$

Testing $-\lambda \Delta u_{\varepsilon}^{\lambda}+B_{\varepsilon}\left(u_{\varepsilon}^{\lambda}\right)+\gamma_{\lambda}\left(u_{\varepsilon}^{\lambda}\right)$ by $\gamma_{\lambda}\left(u_{\varepsilon}^{\lambda}\right)$ and noting that, by monotonicity of $\gamma \lambda$

$$
\begin{aligned}
\int_{\Omega} & \left(-\lambda \Delta u_{\varepsilon}^{\lambda}(t, x)+B_{\varepsilon}\left(u_{\varepsilon}^{\lambda}\right)(t, x)\right) \gamma_{\lambda}\left(u_{\varepsilon}^{\lambda}\right)(t, x) \mathrm{d} x \\
= & \lambda \int_{\Omega} \gamma_{\lambda}^{\prime}\left(u_{\varepsilon}^{\lambda}\right)\left|\nabla u_{\varepsilon}^{\lambda}(t, x)\right|^{2} \mathrm{~d} x \\
& +\frac{1}{2} \int_{\Omega} \int_{\Omega} K_{\varepsilon}(x, y)\left(\gamma_{\lambda}\left(u_{\varepsilon}^{\lambda}(t, x)\right)-\gamma_{\lambda}\left(u_{\varepsilon}^{\lambda}(t, y)\right)\right)\left(u_{\varepsilon}^{\lambda}(t, x)-u_{\varepsilon}^{\lambda}(t, y)\right) \mathrm{d} x \mathrm{~d} y \geqq 0,
\end{aligned}
$$

by the estimate above and the Young inequality we also deduce that

$$
\left\|-\lambda \Delta u_{\varepsilon}^{\lambda}+B_{\varepsilon}\left(u_{\varepsilon}^{\lambda}\right)\right\|_{L^{2}(0, T ; H)}+\left\|\gamma_{\lambda}\left(u_{\varepsilon}^{\lambda}\right)\right\|_{L^{2}(0, T ; H)} \leqq C_{\varepsilon} .
$$




\subsection{Passage to the Limit as $\lambda \searrow 0$}

In this section we analyze the passage to the limit as $\lambda \searrow 0$, with $\varepsilon>0$ and $\tau_{\varepsilon}>0$ still fixed. In view of the uniform bounds (4.8)-(4.12) and the Aubin-Lions lemma, up to the extraction of (not relabeled) subsequences we have the following convergences:

$$
\begin{array}{rr}
u_{\varepsilon}^{\lambda} \rightarrow u_{\varepsilon} & \text { in } C^{0}\left([0, T] ; V^{*}\right), \\
u_{\varepsilon}^{\lambda} \stackrel{*}{\rightarrow} u_{\varepsilon} & \text { in } L^{\infty}\left(0, T ; V_{\varepsilon}\right) \cap H^{1}(0, T ; H), \\
\lambda u_{\varepsilon}^{\lambda} \rightarrow 0 & \text { in } L^{\infty}(0, T ; V), \\
\mu_{\varepsilon}^{\lambda} \rightarrow \mu_{\varepsilon} & \text { in } L^{2}(0, T ; W), \\
\gamma_{\lambda}\left(u_{\varepsilon}^{\lambda}\right) \rightarrow \xi_{\varepsilon} & \text { in } L^{2}(0, T ; H), \\
\Pi\left(u_{\varepsilon}^{\lambda}\right) \rightarrow \Xi_{\varepsilon} & \text { in } L^{2}(0, T ; H), \\
-\lambda \Delta u_{\varepsilon}^{\lambda}+B_{\varepsilon}\left(u_{\varepsilon}^{\lambda}\right) \rightarrow \eta_{\varepsilon} & \text { in } L^{2}(0, T ; H),
\end{array}
$$

for some

$$
\begin{gathered}
u_{\varepsilon} \in H^{1}(0, T ; H) \cap L^{\infty}\left(0, T ; V_{\varepsilon}\right), \quad \mu_{\varepsilon} \in L^{2}(0, T ; W), \\
\xi_{\varepsilon} \in L^{2}(0, T ; H), \quad \Xi_{\varepsilon} \in L^{2}(0, T ; H), \quad \eta_{\varepsilon} \in L^{2}(0, T ; H) .
\end{gathered}
$$

From (4.14) and the fact that $B_{\varepsilon} \in \mathscr{L}\left(V_{\varepsilon}, V_{\varepsilon}^{*}\right)$, it is readily seen that

$$
B_{\varepsilon}\left(u_{\varepsilon}^{\lambda}\right) \stackrel{*}{\rightarrow} B_{\varepsilon}\left(u_{\varepsilon}\right) \quad \text { in } L^{\infty}\left(0, T ; V_{\varepsilon}^{*}\right) .
$$

Moreover, from (4.15) and (4.19), it follows by comparison that

$$
B_{\varepsilon}\left(u_{\varepsilon}^{\lambda}\right) \rightarrow \eta_{\varepsilon} \quad \text { in } L^{2}\left(0, T ; V^{*}\right) .
$$

We deduce in particular that $B_{\varepsilon}\left(u_{\varepsilon}\right)=\eta_{\varepsilon} \in L^{2}(0, T ; H)$, so that also $u_{\varepsilon} \in$ $L^{2}\left(0, T ; W_{\varepsilon}\right)$. The strong convergence (4.13) implies also that $u_{\varepsilon}(0)=u_{0, \varepsilon}$.

Passing to the limit in (4.1)-(4.4) in the weak topology of $L^{2}(0, T ; H)$, we obtain

$$
\begin{aligned}
\partial_{t} u_{\varepsilon}-\Delta \mu_{\varepsilon}=0 & \text { in } L^{2}(0, T ; H), \\
\mu_{\varepsilon}=\tau_{\varepsilon} \partial_{t} u_{\varepsilon}+B_{\varepsilon}\left(u_{\varepsilon}\right)+\xi_{\varepsilon}+\Xi_{\varepsilon}-g_{\varepsilon} & \text { in } L^{2}(0, T ; H), \\
\partial_{\mathbf{n}} \mu_{\varepsilon}=0 & \text { in } L^{2}(\Sigma), \\
u_{\varepsilon}(0)=u_{0, \varepsilon} & \text { in } H .
\end{aligned}
$$

We proceed now providing an identification of the nonlinear terms $\xi_{\varepsilon}$ and $\Xi_{\varepsilon}$ : we adapt an argument performed in [22, Subsection 3.6]. To this end, since $\Pi$ is Lipschitz-continuous, there exists $\alpha>0$ such that the operator

$$
\gamma+\Pi+\alpha \tau_{\varepsilon} \text { Id }: \mathbb{R} \rightarrow 2^{\mathbb{R}}
$$


is maximal monotone. For example, one can choose $\alpha:=\frac{2}{\tau_{\varepsilon}}\left\|\Pi^{\prime}\right\|_{L^{\infty}(\mathbb{R})}$ (recall that $\tau_{\varepsilon}>0$ is fixed). Multiplying (4.2) by $e^{-\alpha t}$, we obtain

$$
\begin{aligned}
e^{-\alpha t} \mu_{\varepsilon}^{\lambda}= & \tau_{\varepsilon} \partial_{t}\left(e^{-\alpha t} u_{\varepsilon}^{\lambda}\right)-\lambda \Delta\left(e^{-\alpha t} u_{\varepsilon}^{\lambda}\right)+B_{\varepsilon}\left(e^{-\alpha t} u_{\varepsilon}^{\lambda}\right)+e^{-\alpha t}\left(\gamma_{\lambda}\left(u_{\varepsilon}^{\lambda}\right)\right. \\
& \left.+\Pi\left(u_{\varepsilon}^{\lambda}\right)+\alpha \tau_{\varepsilon} u_{\varepsilon}^{\lambda}-g_{\varepsilon}\right) .
\end{aligned}
$$

Thus, testing the previous equation by $e^{-\alpha t} u_{\varepsilon}^{\lambda}$ and integrating in time yields

$$
\begin{aligned}
& \limsup _{\lambda \rightarrow 0} \int_{Q} e^{-2 \alpha s}\left(\gamma_{\lambda}\left(u_{\varepsilon}^{\lambda}(s, x)\right)+\Pi\left(u_{\varepsilon}^{\lambda}(s, x)\right)+\alpha \tau_{\varepsilon} u_{\varepsilon}^{\lambda}(s, x)\right) u_{\varepsilon}^{\lambda}(s, x) \mathrm{d} x \mathrm{~d} s \\
& \leq \underset{\lambda \rightarrow 0}{\limsup _{\sin }}\left[\int_{Q} e^{-2 \alpha s} \mu_{\varepsilon}^{\lambda}(s, x) u_{\varepsilon}^{\lambda}(s, x) \mathrm{d} x \mathrm{~d} s-\lambda \int_{Q} e^{-2 \alpha s}\left|\nabla u_{\varepsilon}^{\lambda}(s, x)\right|^{2} \mathrm{~d} x \mathrm{~d} s\right. \\
& \quad-\frac{\tau_{\varepsilon}}{2} \int_{\Omega} e^{-2 \alpha T}\left|u_{\varepsilon}^{\lambda}(T, x)\right|^{2} \mathrm{~d} x+\frac{\tau_{\varepsilon}}{2} \int_{\Omega}\left|u_{0, \varepsilon}^{\lambda}(x)\right|^{2} \mathrm{~d} x \\
& -2 \int_{0}^{T} e^{-2 \alpha s} E_{\varepsilon}\left(u_{\varepsilon}^{\lambda}(s, \cdot)\right) \mathrm{d} s \\
& \left.\quad+\int_{Q} e^{-2 \alpha s} g_{\varepsilon}(s, x) u_{\varepsilon}^{\lambda}(s, x) \mathrm{d} x \mathrm{~d} s\right] .
\end{aligned}
$$

On the one hand, owing to (4.13) and (4.16),

$$
\begin{gathered}
\lim _{\lambda \rightarrow 0} \int_{Q} e^{-2 \alpha s}\left(\mu_{\varepsilon}^{\lambda}(s, x)+g_{\varepsilon}(s, x)\right) u_{\varepsilon}^{\lambda}(s, x) \mathrm{d} x \mathrm{~d} s \\
\quad=\int_{Q} e^{-2 \alpha s}\left(\mu_{\varepsilon}(s, x)+g_{\varepsilon}(s, x)\right) u_{\varepsilon}(s, x) \mathrm{d} x \mathrm{~d} s .
\end{gathered}
$$

On the other hand, by the weak lower semicontinuity of the norms, the convergence (4.14), and the assumption (4.5), we have

$$
\begin{aligned}
\limsup _{\lambda \rightarrow 0} & {\left[-\lambda \int_{Q} e^{-2 \alpha s}\left|\nabla u_{\varepsilon}^{\lambda}(s, x)\right|^{2} \mathrm{~d} x \mathrm{~d} s\right.} \\
& -\frac{\tau_{\varepsilon}}{2} \int_{\Omega} e^{-2 \alpha T}\left|u_{\varepsilon}^{\lambda}(T, x)\right|^{2} \mathrm{~d} x+\frac{\tau_{\varepsilon}}{2} \int_{\Omega}\left|u_{0, \varepsilon}^{\lambda}(x)\right|^{2} \mathrm{~d} x \\
& \left.-2 \int_{0}^{T} e^{-2 \alpha s} E_{\varepsilon}\left(u_{\varepsilon}^{\lambda}(s, \cdot)\right) \mathrm{d} s\right] \\
\leqq & -\frac{\tau_{\varepsilon}}{2} \liminf _{\lambda \rightarrow 0} \int_{\Omega} e^{-2 \alpha T}\left|u_{\varepsilon}^{\lambda}(t, x)\right|^{2} \mathrm{~d} x+\frac{\tau_{\varepsilon}}{2} \limsup _{\lambda \rightarrow 0} \int_{\Omega}\left|u_{0, \varepsilon}^{\lambda}(x)\right|^{2} \mathrm{~d} x \\
& -2 \liminf _{\lambda \rightarrow 0} \int_{0}^{T} e^{-2 \alpha s} E_{\varepsilon}\left(u_{\varepsilon}^{\lambda}(s, \cdot)\right) \mathrm{d} s \\
\leqq & -\frac{\tau_{\varepsilon}}{2} \int_{\Omega} e^{-2 \alpha T}\left|u_{\varepsilon}(T, x)\right|^{2} \mathrm{~d} x+\frac{\tau_{\varepsilon}}{2} \int_{\Omega}\left|u_{0, \varepsilon}(x)\right|^{2} \mathrm{~d} x \\
- & 2 \int_{0}^{T} e^{-2 \alpha s} E_{\varepsilon}\left(u_{\varepsilon}(s, \cdot)\right) \mathrm{d} s .
\end{aligned}
$$


Hence, we deduce that

$$
\begin{aligned}
& \limsup _{\lambda \rightarrow 0} \int_{Q} e^{-2 \alpha s}\left(\gamma_{\lambda}\left(u_{\varepsilon}^{\lambda}(s, x)\right)+\Pi\left(u_{\varepsilon}^{\lambda}(s, x)\right)+\alpha \tau_{\varepsilon} u_{\varepsilon}^{\lambda}(s, x)\right) u_{\varepsilon}^{\lambda}(s, x) \mathrm{d} x \mathrm{~d} s \\
& \leq \int_{Q} e^{-2 \alpha s}\left(\mu_{\varepsilon}(s, x)+g_{\varepsilon}(s, x)\right) u_{\varepsilon}(s, x) \mathrm{d} x \mathrm{~d} s \\
& \quad-\frac{\tau_{\varepsilon}}{2} \int_{\Omega} e^{-2 \alpha T}\left|u_{\varepsilon}(T, x)\right|^{2} \mathrm{~d} x+\frac{\tau_{\varepsilon}}{2} \int_{\Omega}\left|u_{0, \varepsilon}(x)\right|^{2} \mathrm{~d} x \\
& \quad-2 \int_{0}^{T} e^{-2 \alpha s} E_{\varepsilon}\left(u_{\varepsilon}(s, \cdot)\right) \mathrm{d} s .
\end{aligned}
$$

Testing (4.21) by $e^{-2 \alpha t} u_{\varepsilon}$ and integrating in time, the right-hand side of (4.24) rewrites as

$$
\begin{gathered}
\limsup _{\lambda \rightarrow 0} \int_{Q} e^{-2 \alpha s}\left(\gamma_{\lambda}\left(u_{\varepsilon}^{\lambda}(s, x)\right)+\Pi\left(u_{\varepsilon}^{\lambda}(s, x)\right)+\alpha \tau_{\varepsilon} u_{\varepsilon}^{\lambda}(s, x)\right) u_{\varepsilon}^{\lambda}(s, x) \mathrm{d} x \mathrm{~d} s \\
\leqq \int_{0}^{t} \int_{\Omega} e^{-2 \alpha s}\left(\xi_{\varepsilon}(s, x)+\Xi_{\varepsilon}(s, x)+\alpha \tau_{\varepsilon} u_{\varepsilon}(s, x)\right) u_{\varepsilon}(s, x) \mathrm{d} x \mathrm{~d} s .
\end{gathered}
$$

Since the bilinear form

$$
\left(v_{1}, v_{2}\right) \mapsto \int_{Q} e^{-2 \alpha x} v_{1}(s, x) v_{2}(s, x) \mathrm{d} x \mathrm{~d} s, \quad v_{1}, v_{2} \in L^{2}(Q),
$$

is an equivalent scalar product on $L^{2}(Q)$, by the maximal monotonicity of $\gamma+\Pi+$ $\alpha \tau_{\varepsilon}$ Id we conclude that

$$
\xi_{\varepsilon}+\Xi_{\varepsilon}+\alpha \tau_{\varepsilon} u_{\varepsilon} \in\left(\gamma+\Pi+\alpha \tau_{\varepsilon} \text { Id }\right)\left(u_{\varepsilon}\right) \text { a.e. in } Q \text {. }
$$

This allows us to show the further strong convergences

$$
u_{\varepsilon}^{\lambda}(t) \rightarrow u_{\varepsilon}(t) \quad \text { in } H \quad \forall t \in[0, T], \quad u_{\varepsilon}^{\lambda} \rightarrow u_{\varepsilon} \quad \text { in } L^{2}\left(0, T ; V_{\varepsilon}\right) .
$$

Indeed, taking the difference between (4.2) and (4.21), multiplying again by $e^{-\alpha t}$, and testing by $e^{-\alpha t}\left(u_{\varepsilon}^{\lambda}-u_{\varepsilon}\right)$, we get

$$
\begin{aligned}
& \frac{\tau_{\varepsilon}}{2} \int_{\Omega} e^{-2 \alpha t}\left|\left(u_{\varepsilon}^{\lambda}-u_{\varepsilon}\right)(t, x)\right|^{2} \mathrm{~d} x+\lambda \int_{Q_{t}} e^{-2 \alpha s}\left|\nabla u_{\varepsilon}^{\lambda}(s, x)\right|^{2} \mathrm{~d} x \mathrm{~d} s \\
& \quad+2 \int_{0}^{T} e^{-2 \alpha s} E_{\varepsilon}\left(\left(u_{\varepsilon}^{\lambda}-u_{\varepsilon}\right)(s, x)\right) \mathrm{d} s \\
& \quad+\int_{Q_{t}} e^{-2 \alpha s}\left(\gamma_{\lambda}\left(u_{\varepsilon}^{\lambda}\right)+\Pi\left(u_{\varepsilon}^{\lambda}\right)+\alpha \tau_{\varepsilon} u_{\varepsilon}^{\lambda}-\left(\xi_{\varepsilon}+\Xi_{\varepsilon}+\alpha \tau_{\varepsilon} u_{\varepsilon}\right)\right)(s, x)\left(u_{\varepsilon}^{\lambda}-u_{\varepsilon}\right)(s, x) \mathrm{d} x \mathrm{~d} s \\
& =\frac{\tau_{\varepsilon}}{2} \int_{\Omega}\left|u_{0, \varepsilon}^{\lambda}(x)-u_{0, \varepsilon}(x)\right|^{2} \mathrm{~d} x+\int_{Q_{t}} e^{-2 \alpha s}\left(\mu_{\lambda}-\mu\right)(s, x)\left(u_{\varepsilon}^{\lambda}-u_{\varepsilon}\right)(s, x) \mathrm{d} x \mathrm{~d} s \\
& \quad-\lambda \int_{Q_{t}} e^{-2 \alpha s} \Delta u_{\varepsilon}^{\lambda}(s, x) u_{\varepsilon}(s, x) \mathrm{d} x \mathrm{~d} s .
\end{aligned}
$$

We use now the notation $J_{\lambda}^{\gamma}:=(\operatorname{Id}+\lambda \gamma)^{-1}: \mathbb{R} \rightarrow \mathbb{R}$ for the resolvent of $\gamma$. Summing and subtracting $J_{\lambda}^{\gamma}\left(u_{\varepsilon}^{\lambda}\right)$ in the last term on the left-hand side, rearranging 
the terms, and recalling that $u_{\varepsilon}^{\lambda}-J_{\lambda}^{\gamma}\left(u_{\varepsilon}^{\lambda}\right)=\lambda \gamma_{\lambda}\left(u_{\varepsilon}^{\lambda}\right)$, we infer that, for every $t \in[0, T]$,

$$
\begin{aligned}
& \frac{\tau_{\varepsilon}}{2} \int_{\Omega} e^{-2 \alpha t}\left|\left(u_{\varepsilon}^{\lambda}-u_{\varepsilon}\right)(t, x)\right|^{2} \mathrm{~d} x+2 \int_{0}^{T} e^{-2 \alpha s} E_{\varepsilon}\left(\left(u_{\varepsilon}^{\lambda}-u_{\varepsilon}\right)(s, x)\right) \mathrm{d} s \\
& \quad+\int_{Q_{t}} e^{-2 \alpha s}\left(\gamma_{\lambda}\left(u_{\varepsilon}^{\lambda}\right)+\Pi\left(J_{\lambda}^{\gamma}\left(u_{\varepsilon}^{\lambda}\right)\right)\right. \\
&\left.\quad+\alpha \tau_{\varepsilon} J_{\lambda}^{\gamma}\left(u_{\varepsilon}^{\lambda}\right)-\left(\xi_{\varepsilon}+\Xi_{\varepsilon}+\alpha \tau_{\varepsilon} u_{\varepsilon}\right)\right)(s, x)\left(J_{\lambda}\left(u_{\varepsilon}^{\lambda}\right)-u_{\varepsilon}\right)(s, x) \mathrm{d} x \mathrm{~d} s \\
& \leqq \frac{\tau_{\varepsilon}}{2} \int_{\Omega}\left|u_{0, \varepsilon}^{\lambda}(x)-u_{0, \varepsilon}(x)\right|^{2} \mathrm{~d} x+\int_{Q_{t}} e^{-2 \alpha s}\left(\mu_{\lambda}-\mu\right)(s, x)\left(u_{\varepsilon}^{\lambda}-u_{\varepsilon}\right)(s, x) \mathrm{d} x \mathrm{~d} s \\
&\left.-\int_{Q_{t}} e^{-2 \alpha s} B_{\varepsilon}\left(u_{\varepsilon}^{\lambda}(s, x)\right)\right) u_{\varepsilon}(s, x) \mathrm{d} x \mathrm{~d} s \\
&+\int_{Q_{t}} e^{-2 \alpha s}\left(-\lambda \Delta u_{\varepsilon}^{\lambda}(s, x)+B_{\varepsilon}\left(u_{\varepsilon}^{\lambda}(s, x)\right)\right) u_{\varepsilon}(s, x) \mathrm{d} x \mathrm{~d} s \\
&+\int_{Q_{t}} e^{-2 \alpha s}\left(\Pi\left(J_{\lambda}^{\gamma}\left(u_{\varepsilon}^{\lambda}(s, x)\right)\right)-\Pi\left(u_{\varepsilon}^{\lambda}(s, x)\right)\right. \\
&\left.+\alpha \tau_{\varepsilon}\left(J_{\lambda}^{\gamma}\left(u_{\varepsilon}^{\lambda}\right)-u_{\varepsilon}^{\lambda}\right)(s, x)\right)\left(J_{\lambda}\left(u_{\varepsilon}^{\lambda}\right)-u_{\varepsilon}\right)(s, x) \mathrm{d} x \mathrm{~d} s \\
&-\lambda \int_{Q_{t}} e^{-2 \alpha s}\left(\gamma_{\lambda}\left(u_{\varepsilon}^{\lambda}\right)+\Pi\left(u_{\varepsilon}^{\lambda}\right)+\alpha \tau_{\varepsilon} u_{\varepsilon}^{\lambda}-\left(\xi_{\varepsilon}+\Xi_{\varepsilon}+\alpha \tau_{\varepsilon} u_{\varepsilon}\right)\right) \\
&(s,x) \gamma_{\lambda}\left(u_{\varepsilon}^{\lambda}(s, x)\right) \mathrm{d} x \mathrm{~d} s .
\end{aligned}
$$

Recalling that $\gamma_{\lambda}(r) \in \gamma\left(J_{\lambda}^{\gamma}(r)\right)$ for every $r \in \mathbb{R}$, by (4.25) and the monotonicity of the operator $\gamma+\Pi+\alpha \tau_{\varepsilon} \mathrm{Id}$, the third term on the left-hand side is nonnegative. Let us show that the right-hand side converges to 0 , analyzing each term separately. The first two terms on the right-hand side converge to 0 thanks to (4.5), (4.13) and (4.16). Moreover, thanks to (4.14), (4.19), and the fact that $u_{\varepsilon} \in L^{2}\left(0, T ; W_{\varepsilon}\right)$, we have

$-\int_{Q_{t}} e^{-2 \alpha s} B_{\varepsilon}\left(u_{\varepsilon}^{\lambda}(s, x)\right) u_{\varepsilon}(s, x) \mathrm{d} x \mathrm{~d} s \rightarrow-\int_{Q_{t}} e^{-2 \alpha s} B_{\varepsilon}\left(u_{\varepsilon}(s, x)\right) u_{\varepsilon}(s, x) \mathrm{d} x \mathrm{~d} s$ and

$$
\begin{aligned}
& \int_{Q_{t}} e^{-2 \alpha s}\left(-\lambda \Delta u_{\varepsilon}^{\lambda}(s, x)+B_{\varepsilon}\left(u_{\varepsilon}^{\lambda}(s, x)\right)\right) u_{\varepsilon}(s, x) \mathrm{d} x \mathrm{~d} s \\
& \rightarrow \int_{Q_{t}} e^{-2 \alpha s} B_{\varepsilon}\left(u_{\varepsilon}(s, x)\right) u_{\varepsilon}(s, x) \mathrm{d} x \mathrm{~d} s
\end{aligned}
$$

Finally, since $\left(\gamma_{\lambda}\left(u_{\varepsilon}^{\lambda}\right)\right)_{\lambda}$ is bounded in $L^{2}(0, T ; H)$ by $(4.12)$, using the Lipschitzcontinuity of $P i$, the last two terms on the right-hand side can be handled by

$$
\begin{aligned}
& \lambda\left\|\gamma_{\lambda}\left(u_{\varepsilon}^{\lambda}\right)\right\|_{L^{2}(0, T ; H)}\left(\left\|J_{\lambda}^{\gamma}\left(u_{\varepsilon}^{\lambda}\right)\right\|_{L^{2}(0, T ; H)}+\left\|u_{\varepsilon}\right\|_{L^{2}(0, T ; H)} \|\right. \\
& \left.\quad+\left\|\gamma_{\lambda}\left(u_{\varepsilon}^{\lambda}\right)+\Pi\left(u_{\varepsilon}^{\lambda}\right)+\alpha \tau_{\varepsilon} u_{\varepsilon}^{\lambda}-\left(\xi_{\varepsilon}+\Xi_{\varepsilon}+\alpha \tau_{\varepsilon} u_{\varepsilon}\right)\right\|_{L^{2}(0, T ; H)}\right) \leqq C_{\varepsilon} \lambda \rightarrow 0 .
\end{aligned}
$$

Since $t \in[0, T]$ is arbitrary, the strong convergences (4.26) follows. In particular, this readily implies that $\Xi_{\varepsilon}=\Pi\left(u_{\varepsilon}\right)$ and $\xi_{\varepsilon} \in \gamma\left(u_{\varepsilon}\right)$ almost everywhere in $Q$ by the Lipschitz-continuity of $\Pi$ and by the maximal monotonicity of $\gamma$, respectively. 
It is then clear that $\left(u_{\varepsilon}, \mu_{\varepsilon}, \xi_{\varepsilon}\right)$ is a solution to the nonlocal viscous CahnHilliard equation in the sense of (3.11)-(3.16). This completes the proof of the first assertion of Theorem 3.2.

\subsection{Continuous Dependence}

Let $\left(u_{0, \varepsilon}^{1}, g_{\varepsilon}^{1}\right)$ and $\left(u_{0, \varepsilon}^{2}, g_{\varepsilon}^{2}\right)$ satisfy the assumptions (3.9)-(3.10) with $\left(u_{0, \varepsilon}^{1}\right)_{\Omega}=$ $\left(u_{0, \varepsilon}^{2}\right)_{\Omega}$, and let $\left(u_{\varepsilon}^{1}, \mu_{\varepsilon}^{1}, \xi_{\varepsilon}^{1}\right)$ and $\left(u_{\varepsilon}^{2}, \mu_{\varepsilon}^{2}, \xi_{\varepsilon}^{2}\right)$ be any corresponding solutions to (3.11)-(3.16).

We observe that their difference solves

$$
\begin{aligned}
& \partial_{t}\left(u_{\varepsilon}^{1}-u_{\varepsilon}^{2}\right)-\Delta\left(\mu_{\varepsilon}^{1}-\mu_{\varepsilon}^{2}\right)=0 \text { in } Q, \\
& \mu_{\varepsilon}^{1}-\mu_{\varepsilon}^{2}=\tau_{\varepsilon} \partial_{t}\left(u_{\varepsilon}^{1}-u_{\varepsilon}^{2}\right)+B_{\varepsilon}\left(u_{\varepsilon}^{1}-u_{\varepsilon}^{2}\right)+\xi_{\varepsilon}^{1}-\xi_{\varepsilon}^{2}+\Pi\left(u_{\varepsilon}^{1}\right)-\Pi\left(u_{\varepsilon}^{2}\right)-\left(g_{\varepsilon}^{1}-g_{\varepsilon}^{2}\right) \text { in } \mathbf{Q}, \\
& \partial_{\mathbf{n}}\left(\mu_{\varepsilon}^{1}-\mu_{\varepsilon}^{2}\right)=0 \text { in } \Sigma, \\
&\left(u_{\varepsilon}^{1}-u_{\varepsilon}^{2}\right)(0)=0 \text { in } \Omega .
\end{aligned}
$$

By the assumption on the initial data, we have that $\left(u_{\varepsilon}^{1}-u_{\varepsilon}^{2}\right)_{\Omega}=0$. Therefore, we can test the first equation by $\mathcal{N}\left(u_{\varepsilon}^{1}-u_{\varepsilon}^{2}\right)$, the second by $u_{\varepsilon}^{1}-u_{\varepsilon}^{2}$, and take the difference: by performing classical computations we get

$$
\begin{aligned}
\frac{1}{2} \| & \left(u_{\varepsilon}^{1}-u_{\varepsilon}^{2}\right)(t)\left\|_{V^{*}}^{2}+\frac{\tau_{\varepsilon}}{2}\right\|\left(u_{\varepsilon}^{1}-u_{\varepsilon}^{2}\right)(t) \|_{H}^{2}+2 \int_{0}^{t} E_{\varepsilon}\left(u_{\varepsilon}^{1}-u_{\varepsilon}^{2}\right)(s) \mathrm{d} s \\
& +\int_{Q_{t}}\left(\xi_{\varepsilon}^{1}-\xi_{\varepsilon}^{2}\right)(s, x)\left(u_{\varepsilon}^{1}-u_{\varepsilon}^{2}\right)(s, x) \mathrm{d} x \mathrm{~d} s \\
= & \frac{1}{2}\left\|\left(u_{0, \varepsilon}^{1}-u_{0, \varepsilon}^{2}\right)\right\|_{V^{*}}^{2}+\frac{\tau_{\varepsilon}}{2}\left\|\left(u_{0, \varepsilon}^{1}-u_{0, \varepsilon}^{2}\right)\right\|_{H}^{2} \\
& +\int_{Q_{t}}\left(g_{\varepsilon}^{1}-g_{\varepsilon}^{2}-\Pi\left(u_{\varepsilon}^{1}\right)+\Pi\left(u_{\varepsilon}^{2}\right)\right)(s, x)\left(u_{\varepsilon}^{1}-u_{\varepsilon}^{2}\right)(s, x) .
\end{aligned}
$$

The last term on the left-hand side is nonnegative by the monotonicity of $\gamma$. Hence, the continuous-dependence property stated in Theorem 3.2 follows from the Lipschitz-continuity of $\Pi$ and the Gronwall lemma.

\section{Proof of Theorem 3.3}

This section is devoted to study the asymptotic behavior of solutions to the nonlocal viscous Cahn-Hilliard equation as $\varepsilon \searrow 0$. Let us recall that the family of data $\left(u_{0, \varepsilon}, g_{\varepsilon}\right)_{\varepsilon>0}$ are assumed to satisfy (3.17)-(3.20), while $\left(u_{\varepsilon}, \mu_{\varepsilon}, \xi_{\varepsilon}\right)$ is a corresponding solution to (3.11)-(3.16).

\subsection{The Case $\tau>0$}

We consider here the case $\tau>0$, so that $\tau_{\varepsilon} \rightarrow \tau>0$. As a major consequence, this implies that it is not restrictive to assume that

$$
\exists \tau_{*}>0: \quad \tau_{\varepsilon} \geqq \tau_{*} \quad \forall \varepsilon \in\left(0, \varepsilon_{0}\right) .
$$


We test (3.14) by $\mu_{\varepsilon}$, (3.15) by $\partial_{t} u_{\varepsilon}$, take the difference, and integrate on $Q_{t}$ : recalling (3.18) and using the Young inequality, we deduce that

$$
\begin{aligned}
& \int_{Q_{t}}\left|\nabla \mu_{\varepsilon}(s, x)\right|^{2} \mathrm{~d} x \mathrm{~d} s+\tau_{\varepsilon} \int_{Q_{t}}\left|\partial_{t} u_{\varepsilon}(s, x)\right|^{2} \mathrm{~d} x \mathrm{~d} s+E_{\varepsilon}\left(u_{\varepsilon}(t, \cdot)\right) \\
& \quad+\int_{\Omega}(\hat{\gamma}+\hat{\Pi})\left(u_{\varepsilon}(t, x)\right) \mathrm{d} x \\
& \quad \leqq E_{\varepsilon}\left(u_{0, \varepsilon}\right)+\int_{\Omega}(\hat{\gamma}+\hat{\Pi})\left(u_{0, \varepsilon}(x)\right) \mathrm{d} x+\frac{\tau_{\varepsilon}}{2} \int_{Q_{t}}\left|\partial_{t} u_{\varepsilon}(s, x)\right|^{2} \mathrm{~d} x \mathrm{~d} s \\
& \quad+\frac{1}{\tau_{\varepsilon}} \int_{Q_{t}}\left|g_{\varepsilon}(s, x)\right|^{2} \mathrm{~d} x \mathrm{~d} s .
\end{aligned}
$$

Note that $\frac{1}{\tau_{\varepsilon}} \leqq \frac{1}{\tau_{*}}$ by (5.1). Hence, rearranging the terms and using (3.17) we infer that there exists a constant $C>0$, independent of $\varepsilon$, such that

$$
\left\|\nabla \mu_{\varepsilon}\right\|_{L^{2}(0, T ; H)}+\left\|u_{\varepsilon}\right\|_{H^{1}(0, T ; H) \cap L^{\infty}\left(0, T ; V_{\varepsilon}\right)} \leqq C
$$

hence also, by comparison in (3.14),

$$
\left\|\Delta \mu_{\varepsilon}\right\|_{L^{2}(0, T ; H)} \leqq C .
$$

Now, we can proceed as in the previous Sect. 4.2. Since $\left(u_{\varepsilon}\right)_{\Omega}=\left(u_{0, \varepsilon}\right)_{\Omega}$, we can test (3.14) by $\mathcal{N}\left(u_{\varepsilon}-\left(u_{0, \varepsilon}\right)_{\Omega}\right)$, (3.15) by $u_{\varepsilon}-\left(u_{0, \varepsilon}\right)_{\Omega}$, and sum: we obtain, for almost every $t \in(0, T)$,

$$
\begin{aligned}
& \left\langle\partial_{t} u_{\varepsilon}(t), \mathcal{N}\left(u_{\varepsilon}(t)-\left(u_{0, \varepsilon}\right) \Omega_{\Omega}\right)\right\rangle_{V}+\tau_{\varepsilon}\left\langle\partial_{t} u_{\varepsilon}(t), u_{\varepsilon}(t)-\left(u_{0, \varepsilon}\right)_{\Omega}\right\rangle_{V}+2 E_{\varepsilon}\left(u_{\varepsilon}(t, x)\right) \\
& \quad+\int_{\Omega} \xi_{\varepsilon}(t, x)\left(u_{\varepsilon}(t, x)-\left(u_{0, \varepsilon}\right)_{\Omega}\right) \mathrm{d} x \\
& =\int_{\Omega}\left(g^{\varepsilon}(t, x)-\Pi\left(u_{\varepsilon}\right)(t, x)\right)\left(u_{\varepsilon}(t, x)-\left(u_{0, \varepsilon}\right)_{\Omega}\right) \mathrm{d} x .
\end{aligned}
$$

Again, by the estimates already performed, all the terms are bounded in $L^{2}(0, T)$ except

$$
\int_{\Omega} \xi_{\varepsilon}(t, x)\left(u_{\varepsilon}(t, x)-\left(u_{0, \varepsilon}\right)_{\Omega}\right) \mathrm{d} x .
$$

Thanks to assumption (3.19), there are two constants $c, c^{\prime}>0$, independent of $\varepsilon$, such that

$$
\left\|\xi_{\varepsilon}(t, \cdot)\right\|_{L^{1}(\Omega)} \leqq c \int_{\Omega} \xi_{\varepsilon}(t, \cdot)\left(u_{\varepsilon}(t, x)-\left(u_{0, \varepsilon}\right)_{\Omega}\right) \mathrm{d} x+c^{\prime} .
$$

Hence, we deduce that

$$
\left\|\xi_{\varepsilon}\right\|_{L^{2}\left(0, T ; L^{1}(\Omega)\right)} \leqq C,
$$

which implies, by comparison in (3.15), that

$$
\left\|\left(\mu_{\varepsilon}\right)_{\Omega}\right\|_{L^{2}(0, T)} \leqq C .
$$


We deduce that

$$
\left\|\mu_{\varepsilon}\right\|_{L^{2}(0, T ; W)} \leqq C .
$$

Thus, by comparison in (3.15) and by monotonicity of $\gamma$, we obtain that

$$
\left\|B_{\varepsilon}\left(u_{\varepsilon}\right)\right\|_{L^{2}(0, T ; H)}+\left\|\xi_{\varepsilon}\right\|_{L^{2}(0, T ; H)} \leqq C .
$$

By the Aubin-Lions compactness theorem we infer that, up to the extraction of (not relabeled) subsequences, as $\varepsilon \searrow 0$,

$$
\begin{aligned}
u_{\varepsilon} \rightarrow u & \text { in } C^{0}\left([0, T] ; V^{*}\right), \\
u_{\varepsilon} \rightarrow u & \text { in } H^{1}(0, T ; H), \\
B_{\varepsilon}\left(u_{\varepsilon}\right) \eta & \text { in } L^{2}(0, T ; H), \\
\mu_{\varepsilon} \rightarrow \mu & \text { in } L^{2}(0, T ; W), \\
\xi_{\varepsilon} \rightarrow \xi & \text { in } L^{2}(0, T ; H)
\end{aligned}
$$

for some

$$
u \in H^{1}(0, T ; H), \quad \mu \in L^{2}(0, T ; W), \quad \xi, \eta \in L^{2}(0, T ; H) .
$$

We proceed by showing the strong convergence

$$
u_{\varepsilon} \rightarrow u \quad \text { in } C^{0}([0, T] ; H) .
$$

To this end, we show that the sequence $\left(u_{\varepsilon}\right)_{\varepsilon}$ is Cauchy in $C^{0}([0, T] ; H)$. For any arbitrary $\sigma>0$, we apply Lemma 3 with the choice $\delta:=\frac{\sigma}{4 C}$, where $C>0$ is the constant obtained in the estimates above. We deduce that there exists $\bar{\varepsilon}=\bar{\varepsilon}_{\sigma}$ and $C_{\sigma}>0$ such that

$$
\left\|\left(u_{\varepsilon_{1}}-u_{\varepsilon_{2}}\right)(t)\right\|_{H}^{2} \leqq \frac{\sigma}{4 C}\left(E_{\varepsilon_{1}}\left(u_{\varepsilon_{1}}(t)\right)+E_{\varepsilon_{2}}\left(u_{\varepsilon_{2}}(t)\right)\right)+C_{\sigma}\left\|\left(u_{\varepsilon_{1}}-u_{\varepsilon_{2}}\right)(t)\right\|_{V^{*}}^{2}
$$

for every $\varepsilon_{1}, \varepsilon_{2} \in\left(0, \bar{\varepsilon}_{\sigma}\right)$, for every $t \in[0, T]$. Thanks to (5.2), there exists $\tilde{\varepsilon}_{\sigma} \in\left(0, \bar{\varepsilon}_{\sigma}\right)$ such that

$$
\left\|u_{\varepsilon_{1}}-u_{\varepsilon_{2}}\right\|_{C^{0}\left([0, T] ; V^{*}\right)}^{2} \leqq \frac{\sigma}{2 C_{\sigma}} \quad \forall \varepsilon_{1}, \varepsilon_{2} \in\left(0, \tilde{\varepsilon}_{\sigma}\right) .
$$

Hence, taking the supremum in time and using the estimates above we infer that

$$
\begin{aligned}
\left\|u_{\varepsilon_{1}}-u_{\varepsilon_{2}}\right\|_{C^{0}([0, T] ; H)}^{2} & \\
& \leqq \frac{\sigma}{4 C}\left(\left\|E_{\varepsilon_{1}}\left(u_{\varepsilon_{1}}\right)\right\|_{L^{\infty}(0, T)}+\left\|E_{\varepsilon_{2}}\left(u_{\varepsilon_{2}}\right)\right\|_{L^{\infty}(0, T)}\right)+C_{\sigma}\left\|u_{\varepsilon_{1}}-u_{\varepsilon_{2}}\right\|_{C^{0}\left([0, T] ; V^{*}\right)}^{2} \\
& \leqq \frac{\sigma}{4 C}(C+C)+C_{\sigma} \frac{\sigma}{2 C_{\sigma}}=\sigma
\end{aligned}
$$

for every $\varepsilon_{1}, \varepsilon_{2} \in\left(0, \tilde{\varepsilon}_{\sigma}\right)$. Since $\sigma>0$ is arbitrary, we obtain the strong convergence (5.7). 
Now, from (5.7) and the Lipschitz continuity of $\Pi$, it follows that

$$
\Pi\left(u_{\varepsilon}\right) \rightarrow \Pi(u) \quad \text { in } C^{0}([0, T] ; H),
$$

while the strong-weak closure of $\gamma$ readily ensures that $\xi_{\varepsilon} \in \gamma\left(u_{\varepsilon}\right)$ almost everywhere in $Q$.

To conclude the proof of the theorem, it remains to prove additional spatial regularity for $u$ and to provide an identification of $\eta$. First of all, note that since $\left(u_{\varepsilon}\right)_{\varepsilon}$ is bounded in $L^{\infty}\left(0, T ; V_{\varepsilon}\right)$, by the Ponce criterion [51, Theorem 1.2] we have that $u \in L^{\infty}(0, T ; V)$.

Let us identify now the term $\eta$. We first observe that by Lemma 1 there holds $D E_{\varepsilon}=B_{\varepsilon}$ as operators on $V_{\varepsilon}$. Thus, by Lemma 2, and by the continuous inclusion of $V$ into $V_{\varepsilon}$, we deduce

$$
E_{\varepsilon}\left(z_{1}\right)+\left\langle B_{\varepsilon}\left(z_{1}\right), z_{2}-z_{1}\right\rangle_{V_{\varepsilon}^{*}, V_{\varepsilon}} \leqq E_{\varepsilon}\left(z_{2}\right) \quad \forall z_{1}, z_{2} \in V .
$$

Hence, for all $z \in L^{2}(0, T ; V)$ we deduce that

$$
\begin{aligned}
& \int_{0}^{T} E_{\varepsilon}\left(u_{\varepsilon}(t, \cdot)\right) \mathrm{d} t+\int_{0}^{T} \int_{\Omega} B_{\varepsilon}\left(u_{\varepsilon}(t, x)\right)\left(z(t, x)-u_{\varepsilon}(t, x)\right) \mathrm{d} x \mathrm{~d} t \\
& \quad \leqq \int_{0}^{T} E_{\varepsilon}(z(t, \cdot)) \mathrm{d} t .
\end{aligned}
$$

Owing to Lemma 2, and to the dominated convergence theorem, we have

$$
\int_{0}^{T} E_{\varepsilon}(z(t, \cdot)) \mathrm{d} t \rightarrow \frac{1}{2} \int_{0}^{T} \int_{\Omega}|\nabla z(x, t)|^{2} \mathrm{~d} x \mathrm{~d} t .
$$

On the one hand, (5.4) and (5.7) yield

$$
\begin{gathered}
\int_{0}^{T} \int_{\Omega} B_{\varepsilon}\left(u_{\varepsilon}(t, x)\right)\left(z(t, x)-u_{\varepsilon}(t, x)\right) \mathrm{d} x \mathrm{~d} t \\
\rightarrow \int_{0}^{T} \int_{\Omega} \eta(t, x)(z(t, x)-u(t, x)) \mathrm{d} x \mathrm{~d} t .
\end{gathered}
$$

On the other hand, by the Gamma-convergence result in Lemma 2 and by Fatou's Lemma,

$$
\liminf _{\varepsilon \rightarrow 0} \int_{0}^{T} E_{\varepsilon}\left(u_{\varepsilon}(t, \cdot)\right) \mathrm{d} t \geqq \frac{1}{2} \int_{Q}|\nabla u(t, x)|^{2} \mathrm{~d} x \mathrm{~d} t .
$$

Letting $\varepsilon \rightarrow 0$ in (5.8) and recalling that $u \in L^{\infty}(0, T ; V)$, we obtain the inequality $\frac{1}{2} \int_{Q}|\nabla u(t, x)|^{2} \mathrm{~d} x \mathrm{~d} t+\int_{Q} \eta(t, x)(z(t, x)-u(t, x)) \mathrm{d} x \mathrm{~d} t \leqq \frac{1}{2} \int_{Q}|\nabla z(t, x)|^{2} \mathrm{~d} x \mathrm{~d} t$

for every $z \in L^{2}(0, T ; V)$, which in turn implies that $-\Delta u=\eta \in L^{2}(0, T ; H)$. Since $u \in L^{\infty}(0, T ; V)$ and $\Delta u \in L^{2}(0, T ; H)$ in the sense of distributions for 
example, by [41, Thm. 2.27] the normal derivative $\partial_{\mathbf{n}} u \in L^{2}\left(0, T ; H^{-1 / 2}(\partial \Omega)\right)$ is well defined. We infer that, for almost every $t \in(0, T)$ and for every $\varphi \in V$,

$$
\int_{\Omega} \nabla u(t, x) \cdot \nabla \varphi(x) \mathrm{d} x=\int_{\Omega} \eta(t, x) \varphi(x) \mathrm{d} x,
$$

from which it follows that

$$
-\int_{\Omega} \Delta u(t, x) \varphi(x) \mathrm{d} x+\left\langle\partial_{\mathbf{n}} u(t, \cdot), \varphi_{\mid \partial \Omega}\right\rangle_{H^{-1 / 2}(\partial \Omega), H^{1 / 2}(\partial \Omega)}=\int_{\Omega} \eta(t, x) \varphi(x) .
$$

As $-\Delta u=\eta$ in $L^{2}(0, T ; H)$, we infer that

$$
\left\langle\partial_{\mathbf{n}} u(t, \cdot), \varphi_{0}\right\rangle_{H^{-1 / 2}(\partial \Omega), H^{1 / 2}(\partial \Omega)}=0 \quad \forall \varphi_{0} \in H^{1 / 2}(\Omega),
$$

hence $\partial_{\mathbf{n}} u=0$ almost everywhere in $\Sigma$. Now, since we have that $\Delta u \in L^{2}(0, T ; H)$ and $\partial_{\mathbf{n}} u=0 \in L^{2}\left(0, T ; H^{1 / 2}(\partial \Omega)\right)$, by the elliptic regularity result [41, Thm. 3.2] we infer that $u \in L^{2}(0, T ; W)$. Eventually, letting $\varepsilon \searrow 0$ in the equations (3.14)(3.15) we obtain

$$
\partial_{t} u-\Delta \mu=0 \text { in } L^{2}(0, T ; H)
$$

and

$$
\mu=\tau \partial_{t} u-\Delta u+\xi+\Pi(u)-g \text { in } L^{2}(0, T ; H)
$$

This implies that $u$ is a solution to the local Cahn-Hilliard equation according to conditions (3.3)-(3.8), in the viscous case $\tau>0$. This concludes the proof of Theorem 3.3 in the case $\tau>0$.

\subsection{The case $\tau=0$}

We consider here the case $\tau=0$, so that $\tau_{\varepsilon} \rightarrow 0$.

We perform the first estimate as in the previous section: we test (3.14) by $\mu_{\varepsilon}$, (3.15) by $\partial_{t} u_{\varepsilon}$, take the difference, and integrate on $Q_{t}$ : we obtain

$$
\begin{aligned}
& \int_{Q_{t}}\left|\nabla \mu_{\varepsilon}(s, x)\right|^{2} \mathrm{~d} x \mathrm{~d} s+\tau_{\varepsilon} \int_{Q_{t}}\left|\partial_{t} u_{\varepsilon}(s, x)\right|^{2} \mathrm{~d} x \mathrm{~d} s+E_{\varepsilon}\left(u_{\varepsilon}(t, \cdot)\right)+ \\
& \quad \int_{\Omega}(\hat{\gamma}+\hat{\Pi})\left(u_{\varepsilon}(t, x)\right) \mathrm{d} x \\
& \quad=E_{\varepsilon}\left(u_{0, \varepsilon}\right)+\int_{\Omega}(\hat{\gamma}+\hat{\Pi})\left(u_{0, \varepsilon}(x)\right) \mathrm{d} x+\int_{Q_{t}} g_{\varepsilon}(s, x) \partial_{t} u_{\varepsilon}(s, x) \mathrm{d} x \mathrm{~d} s .
\end{aligned}
$$


Using now the additional assumption (3.18) in the case $\tau=0$, we can integrate by parts with respect to time in the last term on the right-hand side and use the Young inequality as

$$
\begin{aligned}
& \int_{Q_{t}} g_{\varepsilon}(s, x) \partial_{t} u_{\varepsilon}(s, x) \mathrm{d} x \mathrm{~d} s \\
& =-\int_{Q_{t}} \partial_{t} g_{\varepsilon}(s, x) u_{\varepsilon}(s, x) \mathrm{d} x \mathrm{~d} s+\int_{\Omega} g_{\varepsilon}(t, x) u_{\varepsilon}(t, x) \mathrm{d} x-\int_{\Omega} g_{\varepsilon}(0, x) u_{0, \varepsilon}(x) \mathrm{d} x \\
& \leq \frac{1}{2}\left\|g_{\varepsilon}\right\|_{H^{1}(0, T ; H)}^{2}+\frac{1}{2} \int_{Q_{t}}\left|u_{\varepsilon}(s, x)\right|^{2} \mathrm{~d} x \mathrm{~d} s+\sigma \int_{\Omega}\left|u_{\varepsilon}(t, x)\right|^{2} \mathrm{~d} x+\frac{1}{4 \sigma}\left\|g_{\varepsilon}(t, \cdot)\right\|_{H}^{2} \\
& \quad+\frac{1}{2}\left\|u_{0, \varepsilon}\right\|_{H}^{2}+\frac{1}{2}\left\|g_{\varepsilon}(0, \cdot)\right\|_{H}^{2}
\end{aligned}
$$

for every $\sigma>0$. Moreover, note that by the generalized Poincaré inequality contained in [51, Theorem 1.1], there exist constants $C>0$ and $\bar{\varepsilon} \in\left(0, \varepsilon_{0}\right)$, independent of $\varepsilon$ and of $t$, such that

$$
\int_{\Omega}\left|u_{\varepsilon}(t, x)-\left(u_{\varepsilon}(t, \cdot)\right)_{\Omega}\right|^{2} \mathrm{~d} x \leqq C E_{\varepsilon}\left(u_{\varepsilon}(t, \cdot)\right) \quad \forall \varepsilon \in(0, \bar{\varepsilon}) .
$$

Since $\left(u_{\varepsilon}\right)_{\Omega}=\left(u_{0, \varepsilon}\right)_{\Omega}$, rearranging the terms and choosing $\sigma>0$ sufficiently small (independently of $\varepsilon$ ), we infer that

$$
\begin{aligned}
& \int_{Q_{t}}\left|\nabla \mu_{\varepsilon}(s, x)\right|^{2} \mathrm{~d} x \mathrm{~d} s+\tau_{\varepsilon} \int_{Q_{t}}\left|\partial_{t} u_{\varepsilon}(s, x)\right|^{2} \mathrm{~d} x \mathrm{~d} s+E_{\varepsilon}\left(u_{\varepsilon}(t, \cdot)\right)+\left\|u_{\varepsilon}(t, \cdot)\right\|_{H}^{2} \\
& \leq C\left(E_{\varepsilon}\left(u_{0, \varepsilon}\right)+\left\|u_{0, \varepsilon}\right\|_{H}^{2}+\int_{\Omega}(\hat{\gamma}+\hat{\Pi})\left(u_{0, \varepsilon}(x)\right) \mathrm{d} x+\left\|g_{\varepsilon}\right\|_{H^{1}(0, T ; H)}^{2}\right) \\
& \quad+\int_{Q_{t}}\left|u_{\varepsilon}(s, x)\right|^{2} \mathrm{~d} x \mathrm{~d} s
\end{aligned}
$$

for a certain $C>0$ independent of $\varepsilon$. Recalling then the assumptions (3.17)-(3.18), the Gronwall lemma yields

$$
\left\|\nabla \mu_{\varepsilon}\right\|_{L^{2}(0, T ; H)}+\left\|u_{\varepsilon}\right\|_{L^{\infty}\left(0, T ; V_{\varepsilon}\right)}+\tau_{\varepsilon}^{1 / 2}\left\|\partial_{t} u_{\varepsilon}\right\|_{L^{2}(0, T ; H)} \leqq C,
$$

hence also, by comparison in (3.14),

$$
\left\|\partial_{t} u_{\varepsilon}\right\|_{L^{2}\left(0, T ; V^{*}\right)} \leqq C .
$$

At this point, we proceed exactly as in the previous Sect. 5.1, and infer that

$$
\left\|\xi_{\varepsilon}\right\|_{L^{2}\left(0, T ; L^{1}(\Omega)\right)} \leqq C,
$$

which implies, by comparison in (3.15), that

$$
\left\|\left(\mu_{\varepsilon}\right)_{\Omega}\right\|_{L^{2}(0, T)} \leqq C .
$$

We deduce then that

$$
\left\|\mu_{\varepsilon}\right\|_{L^{2}(0, T ; V)} \leqq C,
$$


and again, by comparison with (3.15) and by the monotonicity of $\gamma$, that

$$
\left\|B_{\varepsilon}\left(u_{\varepsilon}\right)\right\|_{L^{2}(0, T ; H)}+\left\|\xi_{\varepsilon}\right\|_{L^{2}(0, T ; H)} \leqq C .
$$

The Aubin-Lions theorems ensure then that, up to not relabeled subsequence, as $\varepsilon \searrow 0$,

$$
\begin{aligned}
u_{\varepsilon} \rightarrow u & \text { in } C^{0}\left([0, T] ; V^{*}\right), \\
u_{\varepsilon} \stackrel{*}{\rightarrow} u & \text { in } H^{1}\left(0, T ; V^{*}\right) \cap L^{\infty}(0, T ; H), \\
\tau_{\varepsilon} u_{\varepsilon} \rightarrow 0 & \text { in } H^{1}(0, T ; H), \\
B_{\varepsilon}\left(u_{\varepsilon} \rightarrow \eta\right. & \text { in } L^{2}(0, T ; H), \\
\mu_{\varepsilon} \rightarrow \mu & \text { in } L^{2}(0, T ; V), \\
\xi_{\varepsilon} \rightarrow \xi & \text { in } L^{2}(0, T ; H)
\end{aligned}
$$

for some

$u \in H^{1}\left(0, T ; V^{*}\right) \cap L^{\infty}(0, T ; H), \quad \mu \in L^{2}(0, T ; V), \quad \xi, \eta \in L^{2}(0, T ; H)$.

Arguing as in the previous Sect. 5.1 thanks to the Lemma 3, the convergence (5.10) and the boundedness of $\left(E_{\varepsilon}\left(u_{\varepsilon}\right)\right)_{\varepsilon}$ in $L^{\infty}(0, T)$ imply the strong convergence

$$
u_{\varepsilon} \rightarrow u \quad \text { in } C^{0}([0, T] ; H) .
$$

Hence, by the Lipschitz continuity of $\Pi$ we have

$$
\Pi\left(u_{\varepsilon}\right) \rightarrow \Pi(u) \quad \text { in } C^{0}([0, T] ; H),
$$

while the strong-weak closure of $\gamma$ yields $\xi_{\varepsilon} \in \gamma\left(u_{\varepsilon}\right)$ almost everywhere in $Q$. Moreover, still arguing as in the previous section we obtain that $u \in L^{\infty}(0, T ; V)$, $\eta=-\Delta u$, and $u \in L^{2}(0, T ; W)$ by elliptic regularity.

Passing to the weak limit in (3.14)-(3.15) we obtain then

$$
\partial_{t} u-\Delta \mu=0 \text { in } L^{2}\left(0, T ; V^{*}\right)
$$

and

$$
\mu=-\Delta u+\xi+\Pi(u)-g \text { in } L^{2}(0, T ; H) .
$$

This concludes the proof of Theorem 3.3 also in the case $\tau=0$.

Acknowledgements. E.D and L.T. have been supported by the Austrian Science Fund (FWF) project F 65. E.D. has been funded by the Austrian Science Fund (FWF) project V 662 N32. The research of E.D. has been additionally supported from the Austrian Science Fund (FWF) through the grant I 4052 N32, and from BMBWF through the OeAD-WTZ project CZ04/2019. L.S. has been funded by Vienna Science and Technology Fund (WWTF) through Project MA14-009 and by the Austrian Science Fund (FWF) through project M 2876. The authors are thankful to the anonymous Referee for the careful reading of the paper.

Funding Open access funding provided by Austrian Science Fund (FWF). 
Open Access This article is licensed under a Creative Commons Attribution 4.0 International License, which permits use, sharing, adaptation, distribution and reproduction in any medium or format, as long as you give appropriate credit to the original author(s) and the source, provide a link to the Creative Commons licence, and indicate if changes were made. The images or other third party material in this article are included in the article's Creative Commons licence, unless indicated otherwise in a credit line to the material. If material is not included in the article's Creative Commons licence and your intended use is not permitted by statutory regulation or exceeds the permitted use, you will need to obtain permission directly from the copyright holder. To view a copy of this licence, visit http://creativecommons.org/ licenses/by/4.0/.

Publisher's Note Springer Nature remains neutral with regard to jurisdictional claims in published maps and institutional affiliations.

\section{References}

1. Abels, H.: On a diffuse interface model for two-phase flows of viscous, incompressible fluids with matched densities. Arch. Ration. Mech. Anal. 194(2), 463-506, 2009

2. Abels, H., Bosia, S., Grasselli, M.: Cahn-Hilliard equation with nonlocal singular free energies. Ann. Mat. Pura Appl. (4) 194(4), 1071-1106, 2015

3. Abels, H., Garcke, H., Grün, G.: Thermodynamically consistent, frame indifferent diffuse interface models for incompressible two-phase flows with different densities. Math. Models Methods Appl. Sci., 22(3):1150013, 40 (2012)

4. Barbu, V.: Nonlinear Differential Equations of Monotone types in Banach Spaces. Springer Monographs in MathematicsSpringer, New York 2010

5. BATES, P.W., HAN, J.: The Neumann boundary problem for a nonlocal Cahn-Hilliard equation. J. Differ. Equ. 212(2), 235-277, 2005

6. Bonetti, E., Colli, P., Scarpa, L., Tomassetti, G.: A doubly nonlinear Cahn-Hilliard system with nonlinear viscosity. Commun. Pure Appl. Anal. 17(3), 1001-1022, 2018

7. Bourgain, J., Brezis, H., Mironescu, P.: Another look at Sobolev spaces. In Optimal control and partial differential equations, pages 439-455. IOS, Amsterdam, 2001

8. Bourgain, J., Brezis, H., Mironescu, P.: Limiting embedding theorems for $W^{s, p}$ when $s \uparrow 1$ and applications. J. Anal. Math., 87:77-101. Dedicated to the memory of Thomas H. Wolff (2002)

9. Cahn, J., Hilliard, J.: Free energy of a nonuniform system. I. Interfacial free energy. J. Chem. Phys. 28(2), 258-267, 1958

10. CancÈs, C., Matthes, D., NABet, F.: A two-phase two-fluxes degenerate Cahn-Hilliard model as constrained Wasserstein gradient flow. Arch. Ration. Mech. Anal. 233(2), 837866, 2019

11. Cherfils, L., Gatti, S., Miranville, A.: A variational approach to a Cahn-Hilliard model in a domain with nonpermeable walls. J. Math. Sci. (N.Y.), 189(4):604-636. Problems in mathematical analysis. No. 69 (2013)

12. Cherfils, L., Miranville, A., Zelik, S.: The Cahn-Hilliard equation with logarithmic potentials. Milan J. Math. 79(2), 561-596, 2011

13. Cherfils, L., Petcu, M.: A numerical analysis of the Cahn-Hilliard equation with non-permeable walls. Numer. Math. 128(3), 517-549, 2014

14. Colli, P., Farshbaf-Shaker, M.H., Gilardi, G., Sprekels, J.: Optimal boundary control of a viscous Cahn-Hilliard system with dynamic boundary condition and double obstacle potentials. SIAM J. Control Optim. 53(4), 2696-2721, 2015

15. Colli, P., Fukao, T.: Cahn-Hilliard equation with dynamic boundary conditions and mass constraint on the boundary. J. Math. Anal. Appl. 429(2), 1190-1213, 2015

16. Colli, P., FuKao, T.: Equation and dynamic boundary condition of Cahn-Hilliard type with singular potentials. Nonlinear Anal. 127, 413-433, 2015 
17. Colli, P., Fukao, T.: Nonlinear diffusion equations as asymptotic limits of CahnHilliard systems. J. Differ. Equ. 260(9), 6930-6959, 2016

18. Colli, P., Gilardi, G., Sprekels, J.: On the Cahn-Hilliard equation with dynamic boundary conditions and a dominating boundary potential. J. Math. Anal. Appl. 419(2), 972-994, 2014

19. Colli, P., Gilardi, G., Sprekels, J.: A boundary control problem for the pure CahnHilliard equation with dynamic boundary conditions. Adv. Nonlinear Anal. 4(4), 311325,2015

20. Colli, P., Gilardi, G., Sprekels, J.: A boundary control problem for the viscous Cahn-Hilliard equation with dynamic boundary conditions. Appl. Math. Optim. 73(2), 195-225, 2016

21. Colli, P., Gilardi, G., Sprekels, J.: Distributed optimal control of a nonstandard nonlocal phase field system with double obstacle potential. Evol. Equ. Control Theory 6(1), 35-58, 2017

22. Colli, P., Scarpa, L.: From the viscous Cahn-Hilliard equation to a regularized forward-backward parabolic equation. Asymptot. Anal. 99(3-4), 183-205, 2016

23. Colli, P., Sprekels, J.: Optimal boundary control of a nonstandard Cahn-Hilliard system with dynamic boundary condition and double obstacle inclusions. In Solvability, regularity, and optimal control of boundary value problems for PDEs, volume 22 of Springer INdAM Ser., pp. 151-182. Springer, Cham (2017)

24. Davoli, E., Ranetbauer, H., Scarpa, L., Trussardi, L.: Degenerate nonlocal CahnHilliard equations: well-posedness, regularity and local asymptotics. Ann. Inst. H. Poincaré Anal. Non Linéaire 37, 627-651, 2020

25. Ebenbeck, M., KNOPf, P.: Optimal medication for tumors modeled by a Cahn-HilliardBrinkman equation. Calc. Var. Partial Differential Equations, 58(4) (2019)

26. Frigeri, S., LAM, K.F., RoccA, E.: On a diffuse interface model for tumour growth with non-local interactions and degenerate mobilities. In Solvability, regularity, and optimal control of boundary value problems for PDEs, volume 22 of Springer INdAM Ser., pp. 217-254. Springer, Cham (2017)

27. Gal, C.G., Giorgini, A., Grasselli, M.: The nonlocal Cahn-Hilliard equation with singular potential: well-posedness, regularity and strict separation property. J. Differ. Equ. 263(9), 5253-5297, 2017

28. Gal, C.G., Grasselli, M.: Longtime behavior of nonlocal Cahn-Hilliard equations. Discrete Contin. Dyn. Syst. 34(1), 145-179, 2014

29. Gal, C. G., Grasselli, M., Miranville, A.: Cahn-Hilliard-Navier-Stokes systems with moving contact lines. Calc. Var. Partial Differ. Equ., 55(3):Art. 50, 47 (2016)

30. Gal, C.G., Grasselli, M., Wu, H.: Global weak solutions to a diffuse interface model for incompressible two-phase flows with moving contact lines and different densities. Arch. Ration. Mech. Anal. 234(1), 1-56, 2019

31. Garcke, H., Lam, K.F.: Analysis of a Cahn-Hilliard system with non-zero Dirichlet conditions modeling tumor growth with chemotaxis. Discrete Contin. Dyn. Syst. 37(8), 4277-4308, 2017

32. GarcKe, H., LaM, K.F.: Well-posedness of a Cahn-Hilliard system modelling tumour growth with chemotaxis and active transport. Eur. J. Appl. Math. 28(2), 284-316, 2017

33. Garcke, H., Lam, K.F., Nürnberg, R., SitKa, E.: A multiphase Cahn-Hilliard-Darcy model for tumour growth with necrosis. Math. Models Methods Appl. Sci. 28(3), 525577,2018

34. Garcke, H., Lam, K. F., Rocca, E.: Optimal control of treatment time in a diffuse interface model of tumor growth. Appl. Math. Optim. (2017)

35. Garcke, H., Lam, K.F., SitKa, E., Styles, V.: A Cahn-Hilliard-Darcy model for tumour growth with chemotaxis and active transport. Math. Models Methods in Appl. Sci. 26(06), 1095-1148, 2016

36. Giacomin, G., Lebowitz, J.: Phase segregation dynamics in particle systems with long range interactions. I. Macrosc. Limits. J. Stat. Phys. 87(1), 37-61, 1997 
37. Gilardi, G., Miranville, A., Schimperna, G.: On the Cahn-Hilliard equation with irregular potentials and dynamic boundary conditions. Commun. Pure Appl. Anal. 8(3), 881-912, 2009

38. Gilardi, G., Miranville, A., Schimperna, G.: Long time behavior of the CahnHilliard equation with irregular potentials and dynamic boundary conditions. Chin. Ann. Math. Ser. B 31(5), 679-712, 2010

39. Han, J.: The Cauchy problem and steady state solutions for a nonlocal Cahn-Hilliard equation. Electron. J. Differ. Equ., pp. 113, 9 (2004)

40. Hintermüller, M., Wegner, D.: Distributed optimal control of the Cahn-Hilliard system including the case of a double-obstacle homogeneous free energy density. SIAM J. Control Optim. 50(1), 388-418, 2012

41. Kardestuncer, H., Norrie, D.H. (eds.): Chapters 1-3 in Finite Element Handbook. McGraw-Hill Book Co., New York 1987

42. Lin, F.H.: Some dynamical properties of Ginzburg-Landau vortices. Commun. Pure Appl. Math. 49(4), 323-359, 1996

43. Liv, C., Wu, H.: An energetic variational approach for the Cahn-eilliard Equation with dynamic boundary condition: model derivation and mathematical analysis. Arch. Ration. Mech. Anal. 233(1), 167-247, 2019

44. Maier-PaApe, S., Wanner, T.: Spinodal decomposition for the Cahn-Hilliard equation in higher dimensions: nonlinear dynamics. Arch. Ration. Mech. Anal. 151(3), 187-219, 2000

45. MaZ' ya, V., Shaposhnikova, T.: On the Bourgain, Brezis, and Mironescu theorem concerning limiting embeddings of fractional Sobolev spaces. J. Funct. Anal., 195(2):230238 (2002)

46. MaZ' ya, V., Shaposhnikova, T.: Erratum to: “On the Bourgain, Brezis and Mironescu theorem concerning limiting embeddings of fractional Sobolev spaces" [J. Funct. Anal. 195 (2002), no. 2, 230-238; MR1940355 (2003j:46051)]. J. Funct. Anal., 201(1):298$300(2003)$

47. Melchionna, S., Ranetbauer, H., Scarpa, L., Trussardi, L.: From nonlocal to local Cahn-Hilliard equation. Adv. Math. Sci. Appl. 28(1), 197-211, 2019

48. Melchionna, S., Rocca, E.: On a nonlocal Cahn-Hilliard equation with a reaction term. Adv. Math. Sci. Appl. 24(2), 461-497, 2014

49. Miranville, A., Schimperna, G.: On a doubly nonlinear Cahn-Hilliard-Gurtin system. Discrete Contin. Dyn. Syst. Ser. B 14(2), 675-697, 2010

50. Novick-Cohen, A.: On the viscous Cahn-Hilliard equation. In Material instabilities in continuum mechanics (Edinburgh, 1985-1986), Oxford Sci. Publ., pages 329-342. Oxford Univ. Press, New York (1988)

51. Ponce, A.C.: An estimate in the spirit of Poincaré's inequality. J. Eur. Math. Soc. 6(1), $1-15,2004$

52. Ponce, A.C.: A new approach to Sobolev spaces and connections to $\Gamma$-convergence. Calc. Var. Partial Differ. Equ. 19(3), 229-255, 2004

53. Sandier, E., Serfaty, S.: Limiting vorticities for the Ginzburg-Landau equations. Duke Math. J. 117(3), 403-446, 2003

54. Porta, F.D., Grasselli, M.: Convective nonlocal Cahn-Hilliard equations with reaction terms. Discrete Contin. Dyn. Syst. Ser. B 20(5), 1529-1553, 2015

55. Sandier, E., Serfaty, S.: Gamma-convergence of gradient flows with applications to Ginzburg-Landau. Commun. Pure Appl. Math. 57(12), 1627-1672, 2004

56. SCARPA, L.: Existence and uniqueness of solutions to singular Cahn-Hilliard equations with nonlinear viscosity terms and dynamic boundary conditions. J. Math. Anal. Appl. 469(2), 730-764, 2019

57. Serfaty, S.: Stable configurations in superconductivity: uniqueness, multiplicity, and vortex-nucleation. Arch. Ration. Mech. Anal. 149(4), 329-365, 1999 
LuCa Scarpa \& LaRa Trussardi Institut für Mathematik, University of Vienna, Oskar-Morgenstern-Platz 1, 1090 Vienna Austria.

e-mail: luca.scarpa@univie.ac.at URL: https://mat.univie.ac.at/ scarpa/ e-mail: lara.trussardi@univie.ac.at

URL: https://www.mat.univie.ac.at/ trussardi/

and

Elisa Davoli

Institute of Analysis and Scientific Computing, TU Wien,

Wiedner Hauptstrasse 8-10, 1040 Vienna Austria.

e-mail: elisa.davoli@tuwien.ac.at

URL: https://www.asc.tuwien.ac.at/ edavoli/

(Received August 2, 2019 / Accepted September 14, 2020)

Published online October 4, 2020

(C) The Author(s) (2020) 